\title{
Tracing Domestication and Cultivation of Bananas from Phytoliths: An update from Papua New Guinea
}

\author{
Carol J. Lentfer
}

\section{Research}

\begin{abstract}
There is now good evidence from current banana distributions and genetic analysis that Papua New Guinea and nearby regions have played a key role in the domestication of edible Eumusa and Australimusa bananas. Strong support for this also comes from phytoliths in the archaeobotanical record. Seeds have diagnostic phytoliths which can be used to discriminate between the two main sections of edible bananas, the giant banana, Musa ingens, and Ensete. Therefore, the presence of seed phytoliths and their subsequent disappearance from archaeological assemblages can be used to trace processes of domestication leading to parthenocarpy and sterility. Following loss of viable seeds, banana presence can still be documented from phytolith morphotypes from other plant parts, particularly the volcaniform morphotypes from leaves. Nevertheless, according to several pioneer studies, these are more difficult to differentiate unless they occur in regions where certain species or varieties of bananas are not endemic.
\end{abstract}

This paper reviews results from morphometric and morphotypic analyses of Musaceae phytoliths and briefly introduces the 'New Guinea Banana Project' which builds upon previous analyses. The morphometric database, combined with a comprehensive set of images, facilitates banana phytolith identification and is another step forward in solving the issues surrounding banana dispersal, cultivation and domestication, especially in the Pacific/New Guinea region.

\section{Introduction}

Musa bananas (including plantains) constitute major food staples in the tropics and in terms of gross value of food production they are by far the most important world fruit crop. Furthermore, the banana plant is valued for more than just its fruit (Kennedy 2009): the stem, corm, bract (bell), seeds and flowers can also be eaten; the leaves are used for cooking, wrapping, serving food and for shelter from the sun and rain; fibers from the stem and petioles are used for making string, rope and other cordage for weaving; the sap is used for dye; and, the seeds are used as beads or money (Burkill 1935, Lentfer 2003a, b). Given this multitude of uses, even in their wild state and prior to the development of fleshy, seedless (or nearly seedless) fruit (Lentfer 2003a), it is likely that banana plants would have been recognized as a prized resource and exploited in the past in ways similar to the present. Indeed, this is the picture that is gradually emerging from a host of archaeobotanical, biogeographical, biomolecular and genetic evidence (e.g., Carreel et al. 2002, De Langue \& de Maret 1999, Denham et al. 2003, Kennedy 2008, Mbida et al. 2001, Perrier et al. 2009, Vrydaghs et al. 2009). Complex origins and multiple dispersals for banana cultivars are indicated, but perhaps more striking is the significant role that the New Guinea region has played in the development of the domesticated banana. After a brief review of the evidence for the origins and spread of cultivated bananas, this paper outlines a current research project which expands current banana phy-

\section{Correspondence}

Carol J. Lentfer, School of Social Science, University of Queensland, Queensland, AUSTRALIA.

clentfer20@hotmail.com

Ethnobotany Research \& Applications 7:247-270 (2009)

Published: July 30, 2009 
tolith databases for further assessment of phytolith variation within and between wild and domesticated Musa bananas and Ensete in Papua New Guinea.

\section{Origins and distributions of banana cultivars}

There are several wild species of Musa bananas and closely related Ensete species ranging from Africa (Ensete only) to India, Southeast Asia and as far east as New Guinea and the Solomon Islands (Ensete and Musa). Additionally, there are hundreds of diploid, triploid and polyploid cultivars derived from a few wild species from two sections of bananas (Eumusa and Australimusa) that are cultivated in tropical and subtropical regions worldwide (Argent 1976, Arnaud \& Horry 1997, Daniells et al. 2001, De Langhe et al. 2009, Kennedy 2008, Lentfer 2003a, Pollefeys et al. 2004, Sharrock 1990, Simmonds 1959, 1962, Valmayor 2001, Wong et al. 2001). Phytogeography and genetic evidence shows that the domesticated Australimusa $\mathbf{F e}$ ' $\mathrm{i}$ banana almost certainly originated in the New Guinea region (Jarret et al. 1992, MacDaniels 1947, Simmonds 1959), but the origins of the more commonly known and widely marketed Eumusa cultivars have been more difficult to determine. Based on the distribution of diploid and triploid Eumusa bananas that contain an $\mathrm{A}$ genome derived from either Musa acuminata Colla subspecies banksii (F. Muell.) Simmonds or errans (Blanco) R.V. Valmayor or both (see summary in Table 6, Kennedy 2008:85), it appears that the primary center for the domestication of edible Eumusa section bananas was the Philippines/New Guinea region.

Significantly, a long history of banana manipulation by humans, particularly in the New Guinea region, is indicated by the presence of the banksii A genome in AAB plantains as far afield as Africa and the Pacific. Coupled with the proliferation of diploid AA cultivars in New Guinea, this points to the likelihood of an early dispersal from the New Guinea region in two directions, eastwards into the $\mathrm{Pa}$ cific region and westwards through Island Southeast Asia, Malaysia and across to Africa (Kennedy 2008:85-86). The large number of diploid AA cultivars with both banksii and errans genomes, as well as the absence of the errans genome in the African $A A B$ plantains but its presence together with the banksii genome in the Pacific AAB Maia Maoli plantains are interesting and imply complex species and subspecies interactions within the Philippines/New Guinea regions. This would have involved human diffusion of bananas, probably concurrent with the earliest transfer of the banksii A genome westwards into Island Southeast Asia, and then to mainland Asia and eventually Africa, and also subsequently over an extended period as people expanded eastwards into the Pacific (Kennedy 2008, Perrier et al. 2009).

\section{Archaeobotanical records for bananas}

The archaeobotanical record for bananas is very sparse and is mostly derived from microfossil evidence, partic- ularly phytoliths (reviewed in Denham \& Donohue 2009 and Donohue \& Denham 2009). Evidence from starch shows good promise of adding to this (Lentfer 2009), but currently there is only one confirmed record with a positive identification of banana starch associated with an archaeological deposit from Santa Cruz in the Solomon Islands (Crowther 2009). The only other record comes from the Yuku rock shelter site in the Western Highlands of Papua New Guinea, but this has not been confirmed as banana (Horrocks et al. 2008). Most phytolith records to date have been based on the identification of distinctive volcaniform morphotypes from Musaceae leaves. However, identification beyond the family level has not been successfully attempted in most studies because of the difficulties in discriminating between volcaniform morphotypes from different Musaceae genera, sections and species.

In his pioneering work at Kuk in the Western Highlands of Papua New Guinea, Wilson (1985) used morphometric analyses to discriminate between three sections of bananas and he also identified some Musaceae morphotypes in sediments dated to c. 10,000 cal B.P. as Australimusa. However, these identifications were problematic and inconclusive, partly due to the limited set of comparative reference material analyzed, but also due to the assumption that Eumusa section bananas were introduced into New Guinea from Southeast Asia during the mid-to-late Holocene (e.g., Spriggs 1996). More recently, Lentfer (2003a) found that the seeds from Australimusa, Eumusa, Ingentimusa and Ensete have diagnostic phytolith morphotypes (Figure 1), and was able to confirm the presence of Musa ingens Simmonds - the giant cold-tolerant banana belonging to Section Ingentimusa, Ensete glaucum (Roxb.) Cheesman, and Eumusa section bananas from seed phytolith morphotypes in a similar archaeological context at the Kuk Swamp site (Denham et al. 2003, Lentfer 2003b). The earliest records for Eumusa and Ingentimusa seed phytoliths recovered from palaeochannel fills in this analysis were dated at c. 10,000 cal B.P. Eumusa persisted to the top of the archaeological sequence dated at C. 2500 cal B.P. The earliest date for Ensete seed phytoliths recovered from palaeosurface feature fills was c. 7000-6500 cal B.P. Similar to Eumusa, these persisted to the top of the sequence. Volcaniform leaf phytoliths were also present but were not identified to any particular section or species.

In addition to the Kuk site, Lentfer also confirmed the presence of Eumusa section bananas from diagnostic seed phytoliths at the coastal Lapita site of SAC on Watom Island, East New Britain, Papua New Guinea (Lentfer \& Green 2004), as well as Ensete, Eumusa and Australimusa section bananas at the archaeological site of FIF/4 at the Yombon airstrip in South West New Britain (Lentfer et al. 2008). As with Kuk, volcaniform leaf phytoliths were present in both assemblages but no attempt was made to identify them beyond the family level in the initial analyses. 
Lentfer - Tracing Domestication and Cultivation of Bananas from Phytoliths: 249 An update from Papua New Guinea
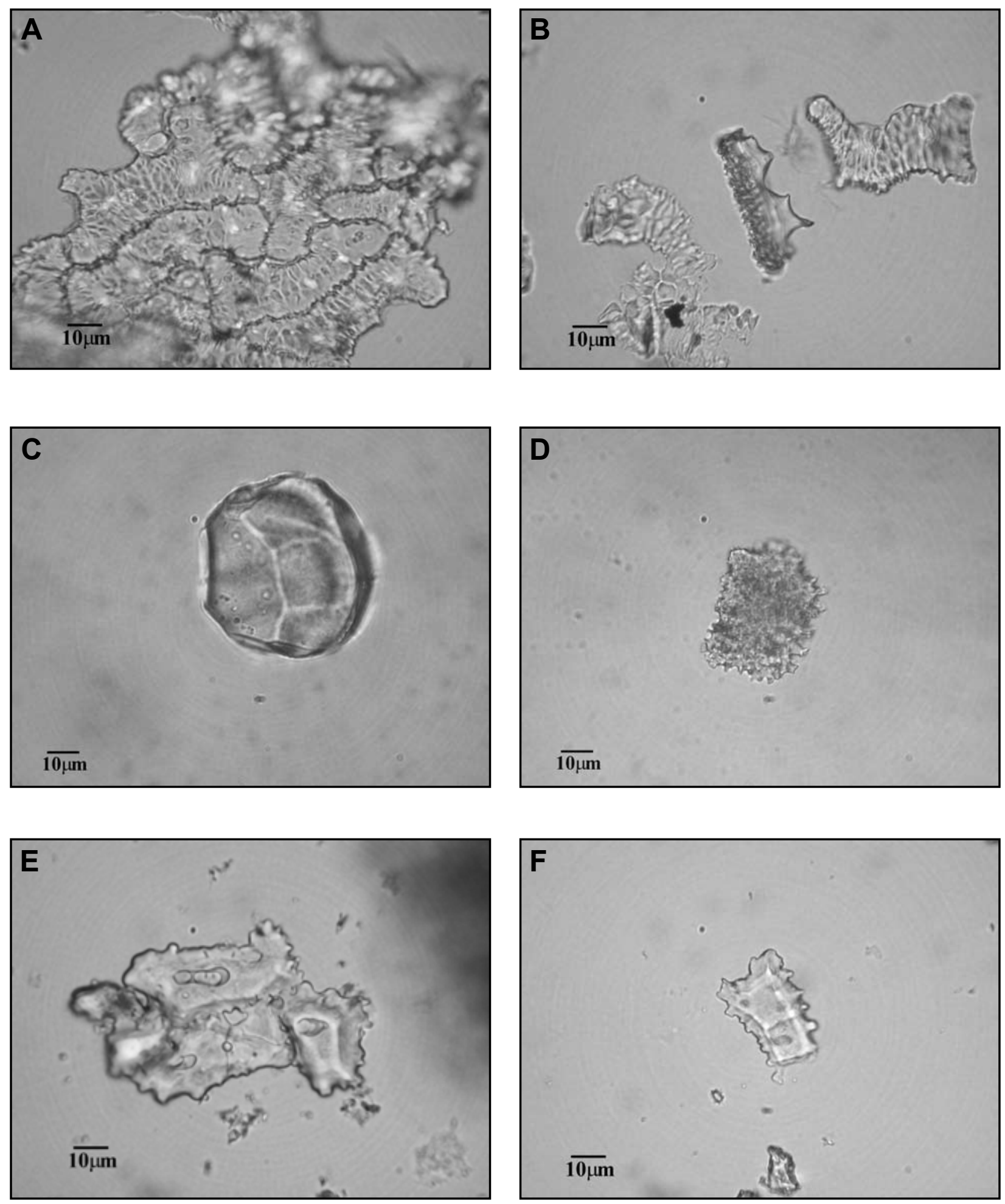

Figure 1. Diagnostic seed morphotypes of wild Musa bananas and Ensete from Papua New Guinea. A-B. Ensete glaucum (Accession No. QH28807); C-D. Musa ingens, Section Ingentimusa (Accession No. WH1); E-F. Musa peekelii, Section Australimusa (Accession No. WNB488). 

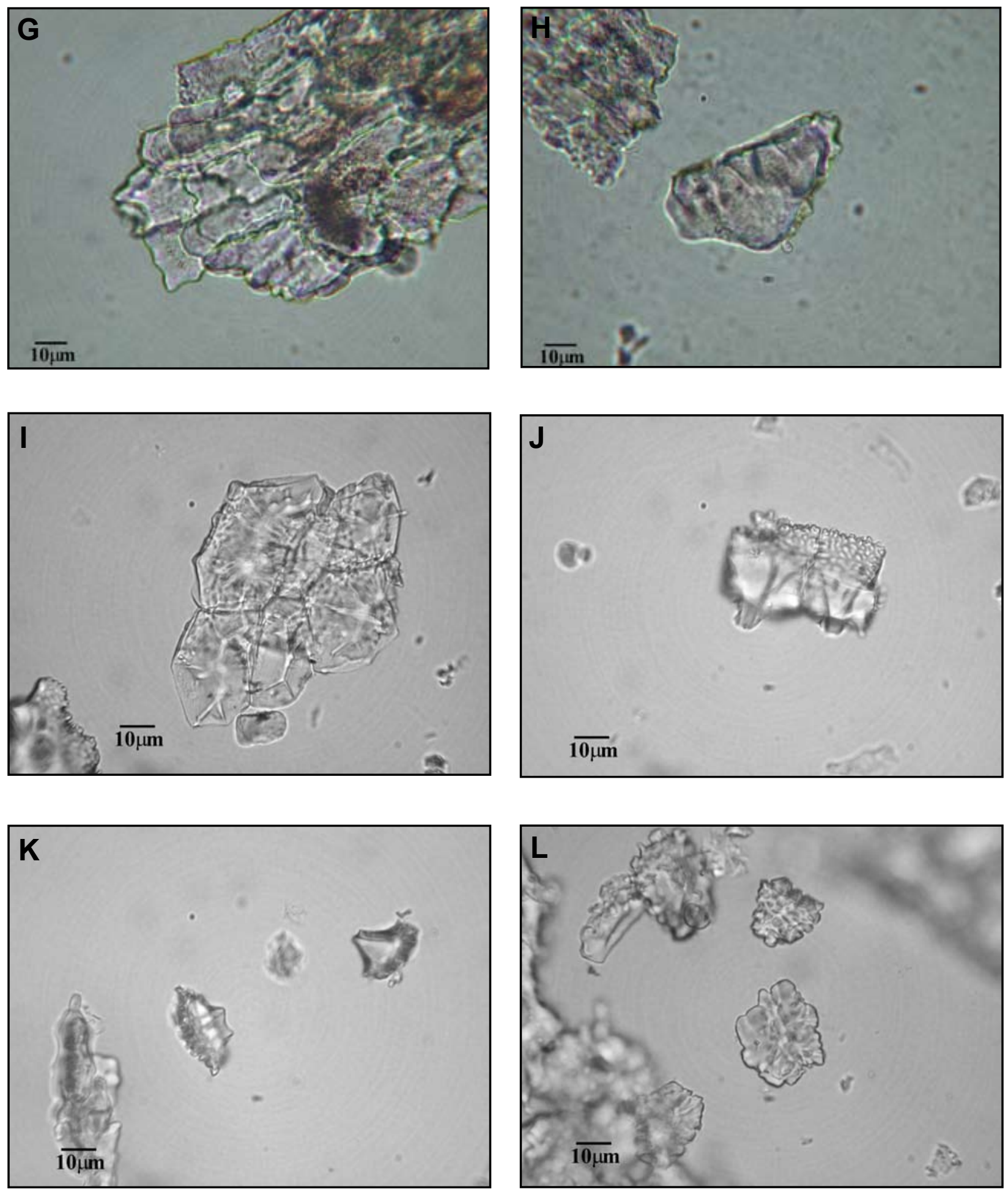

Figure 1 cont. Diagnostic seed morphotypes of wild Musa bananas and Ensete from Papua New Guinea. G-H. Musa maclayi, Section Australimusa (Accession No. MB6); I-J. Musa acuminata ssp.banksii, Section Eumusa (Accession No. QH067962); K-L. Musa schizocarpa, Section Eumusa (Accession No. NB489). 


\section{Lentfer - Tracing Domestication and Cultivation of Bananas from Phytoliths: 251 An update from Papua New Guinea}

Apart from phytolith studies in New Guinea there are few other published accounts of banana identification beyond the family level with the exception of two African studies in Cameroon and Uganda. Volcaniform morphotypes recovered from refuse pits at an agricultural village site, Nkang in southern Cameroon dating to c. 2500 cal B.P. were identified to genus Musa (Mbida et al. 2001, 2004). Further to the east, at Munsa, Uganda, both Musa and Ensete phytoliths were identified from swamp sediment cores (Lejju et al. 2006). The oldest dates for these were c. 5200 cal B.P. (cf. Neumann \& Hildebrand 2009). In contrast to New Guinea, no seed phytoliths were recorded from either African site, probably because Musa bananas were already seedless by the time they had been introduced into Africa.

\section{The question of cultivation}

Banana plants in their natural state are light-demanding pioneer species of tropical environments. Growing naturally from seed and via suckers, bananas are adapted to opportunistic colonization of mostly well-drained open sites such as forest margins, forest gaps resulting from tree fall, and scree slopes associated with landslides and erosion (e.g., Argent 1976, MacDaniels 1947). Human selection, which eventually led to female sterility, loss of seeds and parthenocarpy, has produced hundreds of different land races and hybrids of Eumusa, Australimusa and Eumusa $x$ Australimusa section bananas occurring in the Indo-Pacific region and Africa (see De Langhe et al. 2009) and implies a long history of somatic mutation and human manipulation involving cultivation.

Nevertheless, given the sparse record of prehistoric banana distribution, tracking evidence for cultivation and dispersal of cultivars is difficult. The record is derived mostly from two broad categories of evidence: ecological and geographical. This is mostly reliant on archaeobotanical finds showing presence of bananas: outside their natural range; in contexts with archaeological, sedimentary and ecofactual features indicative of cultivation; or, in association with other known domesticates and associated species, plants and/or animals. At Kuk, for instance, the altitude is exceptionally high (>1560 metres above sea level) for wild Eumusa section bananas and Ensete to occur naturally (see Argent 1976), but even if the early Holocene environment was warmer than it is today, the presence of stake holes, post holes and mounds, coupled with the relatively high proportions of Musaceae phytoliths in the phytolith assemblages, particularly following erosion and burning episodes, are strongly supportive of human influences and cultivation at least by about $7000-6500$ cal B.P. (Denham et al. 2003).

Evidence for cultivation at the Watom site is equally strong. Banana phytoliths are found in black, humic rich soils typical of gardens. They are in association with phytoliths and macrobotanical remains from other cultivars including co- conut, Canarium, Job's tears, possibly sugar cane, phytoliths derived from pioneer tree species and grasses that colonise gardens, and pig and chicken bones (Lentfer \& Green 2004). All other evidence for cultivation primarily relies on bananas being outside their natural range of distribution. For instance, bananas are outside their natural range in the Pacific east of the Solomon Islands. Therefore, all records for bananas east of the Solomons are indicative of human translocation and cultivation including wild $M$. acuminata ssp. banksii found in Samoa (De Langhe 2009, MacDaniels 1947) and the Musa found associated with Lapita deposits in Vanuatu (Horrocks et al. in press). The same applies in Africa where only Ensete species are indigenous. Musa banana cultivation can be inferred at the Nkang site in Cameroon by 2500 cal B.P. from the presence of Musa phytoliths (Mbida et al. 2001, 2004) and pending the accuracy of dating and the morphotypic discrimination between Musa and Ensete volcaniform phytoliths (Neumann \& Hildebrand 2009), it is possible that banana cultivation occurred in Uganda as early as c. 5200 cal B.P. (Lejju et al. 2006).

Identifying banana cultivation in areas where wild bananas grow naturally is perhaps the most difficult, especially in the absence of supportive archaeological and/or palaeobotanical evidence. At the Yombon airstrip locale, south West New Britain, Papua New Guinea, for instance, bananas appear in the early to middle Holocene. However, although this coincides with a major burning episode, there is no other evidence supportive of cultivation other than the presence of a few potential cultivars including Saccharum sp. and Coix lachryma-jobi L. In cases such as this, cultivation could only be confirmed if it could be proven that bananas were indeed seedless, and therefore cultivars.

\section{Identification of cultivars: morphometric and morphotypic analyses of phytoliths}

Tracing the history of banana cultivation, domestication and dispersal could be greatly facilitated if phytoliths can be readily differentiated in archaeobotanical assemblages. However, the variation of phytoliths within and between Musaceae taxa and within and between plant parts (with the exception of banana seed phytoliths) has, until recently, been poorly understood. To address this shortfall and to expand previous morphotypic and morphometric analyses undertaken by Wilson (1985) and Mbida et al. (2001), phytolith researchers have commenced a series of rigorous morphometric analyses of banana phytoliths to determine their diagnostic value (e.g., Ball et al. 2006).

Lentfer (2003a) has undertaken preliminary studies to investigate the variation of phytoliths within and between species and also within and between plant parts. The first set of exploratory analyses examined seventeen acces- 
Table 1. Banana accessions analysed by Lentfer (2003a, b).

\begin{tabular}{|l|l|l|}
\hline Section & Wild species & $\begin{array}{l}\text { Accession code } \\
\text { (plant parts*) }\end{array}$ \\
\hline \multirow{5}{*}{ Eumusa } & $\begin{array}{l}\text { Musa acuminata } \\
\text { ssp. banksii }\end{array}$ & $\begin{array}{l}\text { QH325354 } \\
\text { (If,sd,sk,mrb,ped); } \\
\text { QH541190 (sd,br) }\end{array}$ \\
\cline { 2 - 3 } & Musa schizocarpa & $\begin{array}{l}489 \text { (If,sd,sk); } \\
\text { QH356650 (If) }\end{array}$ \\
\hline Australimusa & Musa peekelii & $\begin{array}{l}\text { QH067966 } \\
\text { (If,sd); } 488 \text { (If;sd/ } \\
\text { fr); 489 (fr/sd); } \\
\text { QH067968 (sk) }\end{array}$ \\
\cline { 2 - 3 } & & $\begin{array}{l}\text { QH537000 (If,br); } \\
\text { NB487 (If,Ifbs/st); } \\
\text { QH356648(mrb) }\end{array}$ \\
\cline { 2 - 3 } & Musa maclayi & $\begin{array}{l}\text { QH28807 (sd,sk); } \\
482 \text { (If,sk,sd); } \\
\text { QH356652 (If) }\end{array}$ \\
\hline N/A & Ensete glaucum & \\
\hline \multirow{2}{*}{ Eumusa } & Musa acuminata & QH438477 (If) \\
\cline { 2 - 3 } & Musa paradisiaca? & QH4000037 (If) \\
\hline Australimusa & TT(Fe`i) & QH067969 (If) \\
\hline
\end{tabular}

*If=leaf; sd=seed; fr=fruit; sk=skin; br=bract; $\mathrm{mrb}=$ mid rib; st=stem; Ifbs=leaf base; ped=peduncle Note $\mathbf{F e}{ } i$ is referred to as $M$. fei F. Muell. in figures.

sions consisting of Ensete and wild and cultivated Australimusa and Eumusa Section bananas (Table 1). Twenty-five phytoliths from a number of different plant parts including leaf blades, leaf mid-ribs, leaf bases, fruit and seed, skin, pseudostems, bracts and peduncles were analyzed separately. Only phytoliths with craters (i.e., spherical to sub-spherical to blocky morphotypes but not necessarily volcaniform morphotypes, see Figure 2) were included in the analysis. It did not include any of the clearly diagnostic seed morphotypes referred to previously and shown in Figure 1.

\section{Analysis 1}

a) Differentiation of phytolith seed morphotypes from other plant parts: Long dimensions of phytolith bodies and craters were measured (see Figure 1, Vrydaghs et al., 2009) and ratios of body length to crater width were calculated. Results of the analysis using pooled data show that the ratio of mean body length to mean crater width is significantly different at $\alpha=0.05$, differentiating between seed/fruit pulp morphotypes and morphotypes from other plant parts (Figure 3). Notably, body length and crater width scores by themselves were less helpful in this regard.

b) Differentiation of Eumusa, Australimusa seed morphotypes and Ensete: Ensete glaucum has distinctive
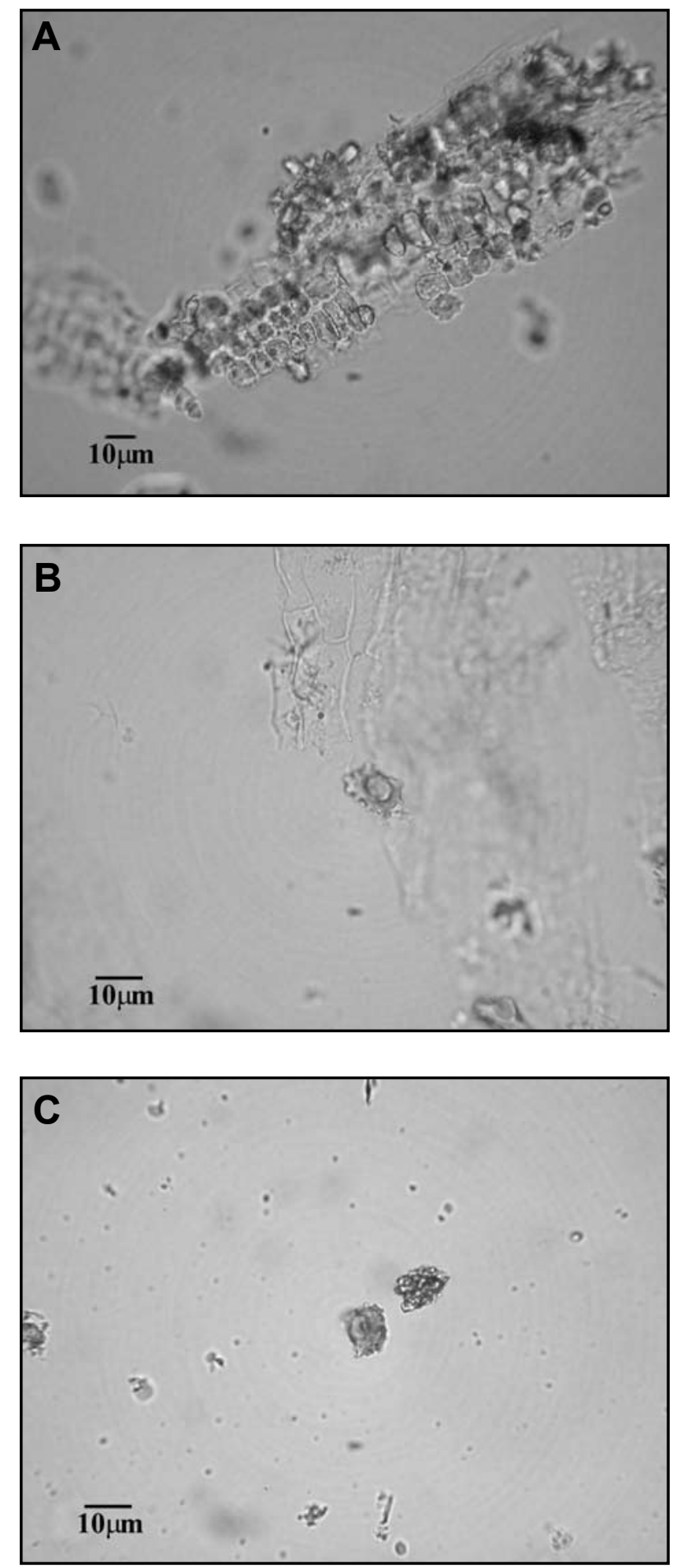

Figure 2. A. Sheet of polygonal and globular seed phytoliths from Musa acuminata ssp. banksii. These morphotypes have craters and were included in the analysis. The plant material was obtained from the Queensland Herbarium (Accession No. QH067962). B-C. Examples of volcaniform and globular leaf morphotypes from Musa maclayi (Accession No. NB487) examined in the analysis. 

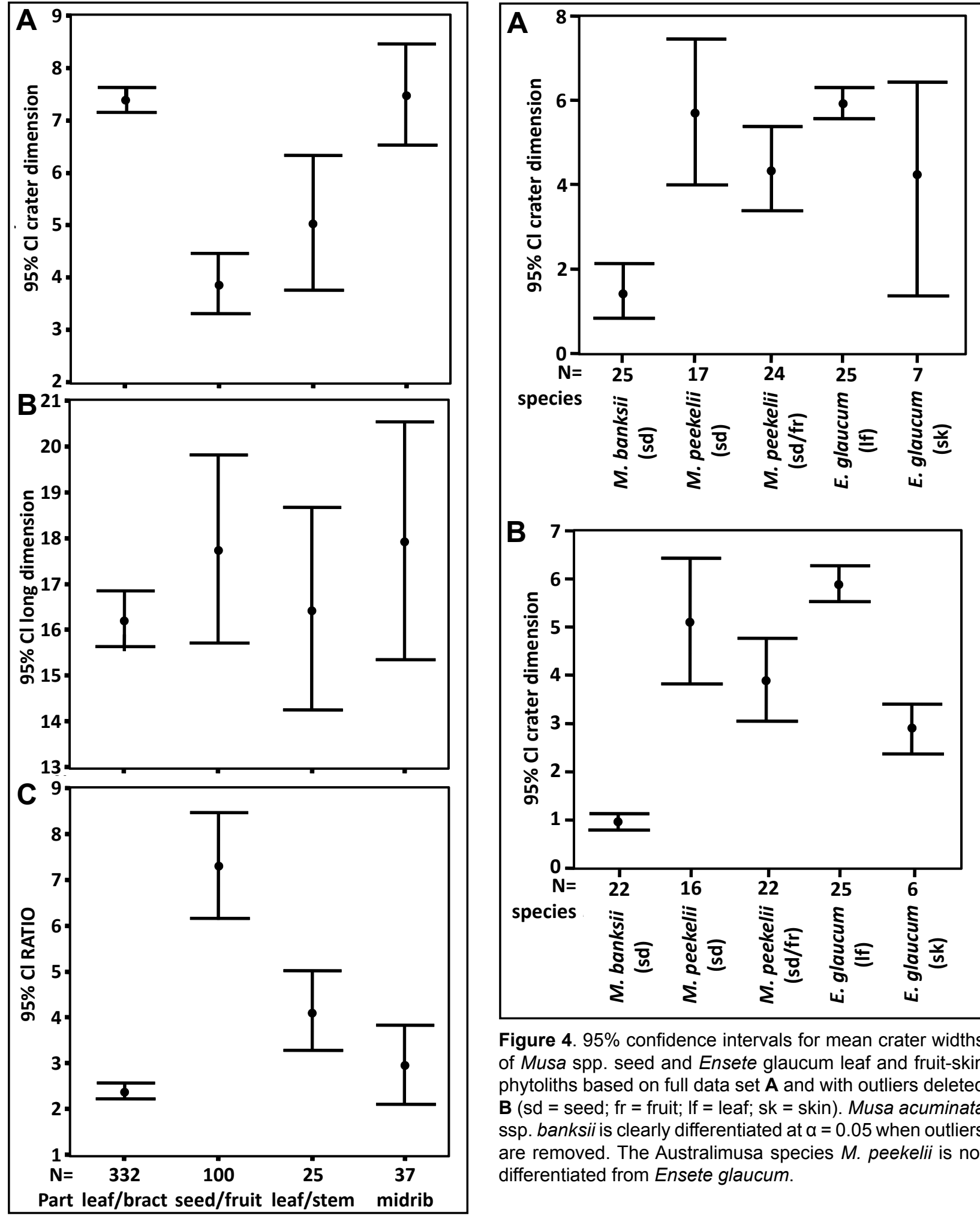

Figure 4. 95\% confidence intervals for mean crater widths of Musa spp. seed and Ensete glaucum leaf and fruit-skin phytoliths based on full data set $\mathbf{A}$ and with outliers deleted B (sd = seed; fr = fruit; If = leaf; sk = skin). Musa acuminata ssp. banksii is clearly differentiated at $\alpha=0.05$ when outliers are removed. The Australimusa species $M$. peekelii is not differentiated from Ensete glaucum.

Figure 3. 95\% confidence intervals for mean crater widths A, mean long dimensions B and mean long dimension/ mean crater width ratios $\mathbf{C}$ of all phytoliths examined. Note that the leaf/stem sample consists of the base of leaf and pseudostem samples and the seed/fruit samples consist of: A) the fruit pulp attached to seeds, and B) seeds. Fruit pulp does not contain phytoliths and therefore phytoliths examined in the analysis are derived from seeds only. The ratio plot (C) shows that seed and seed/fruit phytoliths have significantly smaller craters compared to body length than leaf phytoliths and can be discriminated at $\alpha=0.05$ regardless of species derivation. 
seed morphotypes (see Figure 1) that were not included in this analysis. However, the leaf and fruit skin phytolith morphotypes of Ensete have globular and polygonal morphotypes similar to Musa seed morphotypes. An analysis comparing mean body length and mean crater width of M. acuminata ssp. banksii and Musa peekelii Lauterb. seed morphotypes, and E. glaucum leaf and fruit skin morphotypes showed that the width of craters in $M$. acuminata ssp. banksii are significantly smaller than both $M$. peekelii and Ensete at $\alpha=0.05$ (Figure 4). $M$. peekelii and E. glaucum could not be differentiated according to crater width. However, mean body length of $M$. peekelii was significantly greater at $\alpha=0.05$ than M. acuminata ssp. banksii and Ensete (Figure 5).

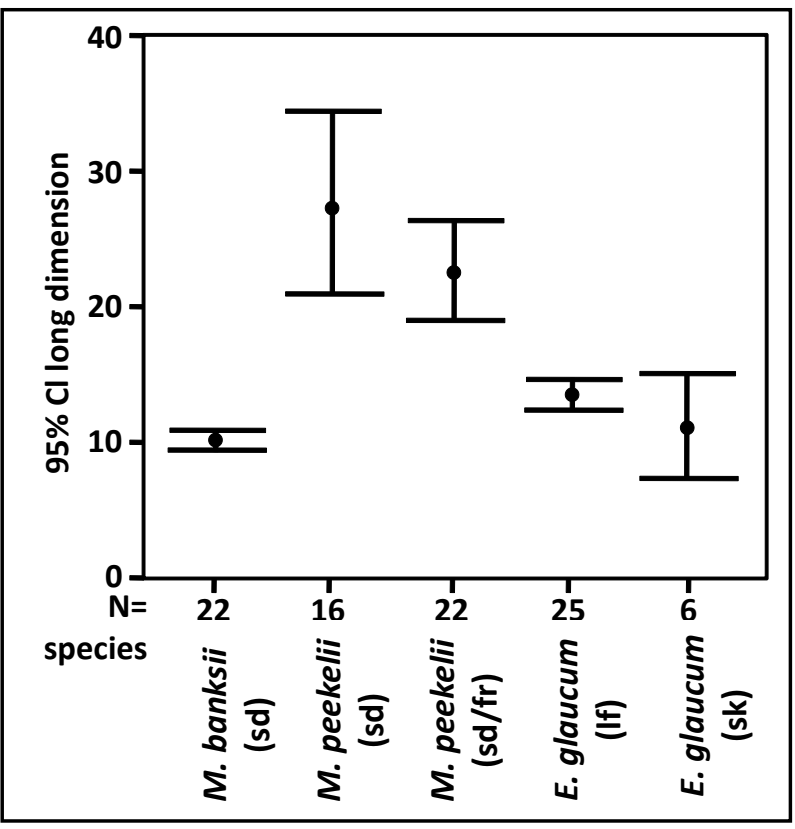

Figure 5. 95\% confidence intervals for mean long dimensions of Musa spp. seed and Ensete leaf and fruitskin phytoliths based on full data set (sd = seed; $\mathrm{fr}=$ fruit; If = leaf; sk = skin). The Australimusa species, $M$. peekelii is differentiated from Musa acuminata ssp. banksii and Ensete glaucum.

\section{Analysis 2}

This analysis was based on morphotypic analysis of the same set of leaf/bract volcaniform morphotypes used in the first analysis. Seven major categories of attributes (Table 2) were examined. Attributes within each category were given a score of 1 if present and 0 if absent and statistically tested using principal components analysis. Similar to the morphometric analysis, Ensete glaucum morphotypes were clearly differentiated by body texture and crater rim characteristics (Figure 6). Other taxa could not be clearly differentiated.

\section{Analysis 3}

Additional morphometric analyses were undertaken to determine if leaf phytolith morphotypes could be further differentiated. Mean body length and mean crater width of leaf/bract volcaniform morphotypes from different taxa were compared (Figures 7 to 9). Crater width of E. glaucum morphotypes was significantly smaller at $\alpha$ $=0.05$ than all other Eumusa and Australimusa bananas with the exception of the wild Australimusa species, Musa maclayi F. Muell. ex Mikl.-Maclay and the wild Eumusa species Musa schizocarpa Simmonds (Figure 7). Body length was significantly smaller than the two $M$. acuminata cultivars (listed as $M$. acuminata and Musa paradisiaca L.), M. maclayi and Fe'i (Figure 8). Crater length and body length of morphotypes from other bananas were more similar and consequently these taxa were found to be more difficult to differentiate (Figures 7 and 8). Crater width of the wild $M$. maclayi morphotypes were significantly different from the cultivated bananas, M. acuminata and Fe $\mathbf{i}$ and the other wild Australimusa banana $M$. peekelii, but overlapped with the wild Eumusa species ( $M$. acuminata ssp. banksii and $M$. schizocarpa). Musa schizocarpa could only be differentiated from the three cultivars ( $M$. acuminata, $M$. paradisiaca and $\mathbf{F e}{ }^{\mathbf{i}}$ ), and the wild $M$. acuminata ssp. banksii was differentiated from only one of the Eumusa section cultivars, M. acuminata, not $M$. paradisiaca. Body length was a less helpful criterion for differentiating taxa than crater width. Interestingly, the only significant difference at $\alpha=0.05$ was between Australimusa section banan-

Table 2. Categories and attributes used in analysis of volcaniform leaf phytoliths (attributes shown in Figure 6B are the abbreviated forms shown in (...) and are equivalent terms from Madella et al. 2005).

\begin{tabular}{|c|c|}
\hline Categories & Attributes \\
\hline Crater & round [r] (orbicular), oval [o], square [sqt], irregular [irrt] \\
\hline Morphology & tabular [t], blocky [b], spherical [sph] (globose), platy [pl] (planar) \\
\hline Base shape & $\begin{array}{l}\text { square [squb], rectangular [rb], quadrilateral }[\mathrm{qb}] \text {, triangular [tb], boat [bb] (oblong), round [cb] } \\
\text { (orbicular), other [ob] }\end{array}$ \\
\hline Height & short [fh] ( $h<1 / 3$ length), medium [mh] ( $h=1 / 3$ to $1 / 2$ length), tall [th] ( $h \geq 1 / 2$ length) \\
\hline Texture & psilate [stx], rough [rtx] verrucate, granulate [grx], dimpled [dtx] \\
\hline $\operatorname{Rim}$ & present [rp]/absent [ra], regular [regr]/irregular [irrr] \\
\hline Ornamentation & absent [no], short [sho], medium [mo], long [lo], lobed [lbo] \\
\hline
\end{tabular}




\section{Lentfer - Tracing Domestication and Cultivation of Bananas from Phytoliths: 255 An update from Papua New Guinea}

as. Body lengths of M. maclayi and Fe i bananas were significantly greater than $M$. peekelii. Finally the ratio of mean body length to crater width differentiated $M$. maclayi from all other samples with the exception of M. schizocarpa (Figure 9). Musa schizocarpa exhibited

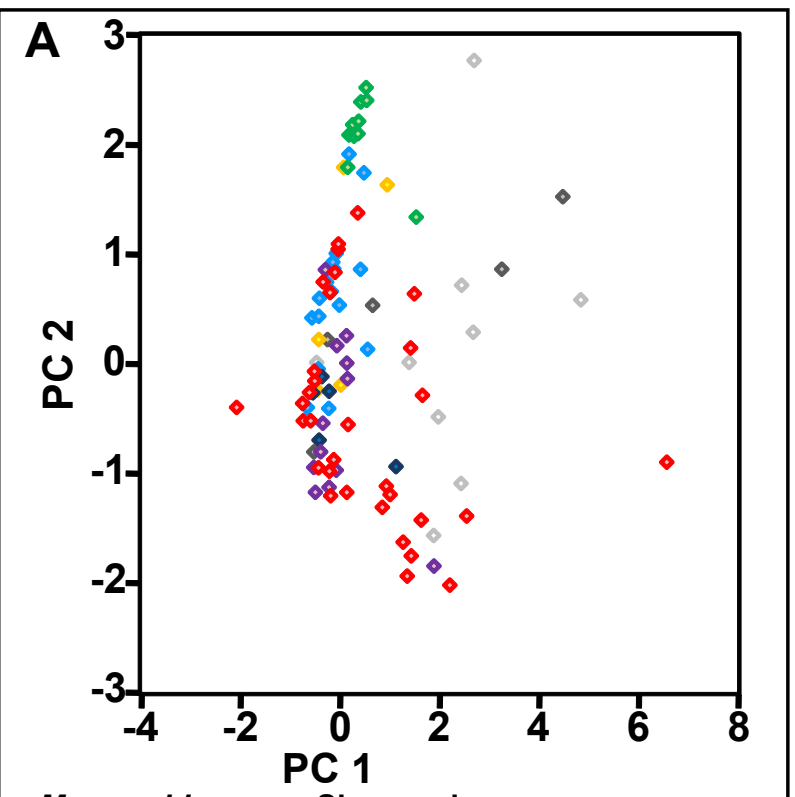

- Musa schizocarpa Simmonds

- Musa peekelii Lauterb.

Musa paradisiaca L.

- Musa maclayi F. Muell. ex Mikl.-Maclay

- Musa fei F. Muell.

- Musa acuminata Colla

- Ensete sp.

- Musa banksii F. Muell.

Figure 6. Biplot of principal components analysis of banana leaf phytolith attribute data. The sample plot $A$ shows that Ensete glaucum is differentiated from other bananas. The main attributes separating it from other bananas are the irregular rim and the rough texture and to a lesser extent, short ornamentation (SHO). See the distribution of attributes in the vector plot $B$. (see abbreviations in Table 2) the greatest variability and could not be differentiated from any other taxon.

Lentfer's findings broadly concur with those of Ball et al. (2006) and Vrydaghs et al. (2009), which have been confined to the distinctive volcaniform leaf phytoliths from a different set of Musaceae accessions including M. acuminata, Musa balbisiana Colla and various cultivar groups. A combination of morphometric (base length and crater width) and morphotypic analyses (base shape, crater position and cone shape) (Ball et al. 2006:3) can help to discriminate between certain taxa. Notably, wild diploid $M$. balbisiana (BB) volcaniform morphotypes were found to be significantly larger than both wild and edible diploid M. acuminata (AA) morphotypes (Ball et al. 2006:7), but edible AA could not be differentiated from wild AA. Subsequent studies analyzing $A A, A A A, A A B$ and $A B B$ have found a very complex pattern of phytolith variation. Continuing analyses with additional samples are further investigating the variation in crater width, particularly the role of banksii alleles in its expression (Vrydaghs et al. 2009).

\section{Implications for future research}

The presence of seeded bananas in archaeobotanical assemblages can be identified from seed phytolith morphotypes. Distinctive morphotypes shown in Figure 1 are diagnostic at the section level. Other globular and polygonal morphotypes can be differentiated from other plant parts by body length/crater width ratios. Additionally, preliminary studies indicate that Eumusa bananas ( $M$. acuminata ssp. banksii) can be differentiated from Australimusa bananas ( $M$. peekelii) on the basis of crater width and body 
length. Although further comparative studies are needed to include a range of other species from either section, current results suggest the outlook is very promising for tracking the complex history of Musaceae in the archaeobotanical record. Phytoliths can be used to identify natural distributions of Musa and Ensete, differentiate wild populations from fully domesticated (seedless) populations and trace patterns of dispersal. However, based on this premise, mixed populations of wild and cultivated bananas (a common occurrence in Papua New Guinea; Lentfer 2003b; Jean Kennedy pers. comm.) and partially domesticated populations prior to the complete loss of seed - for

Figure $7.95 \%$ confidence intervals for mean crater widths of leaf phytoliths differentiating Ensete glaucum from all other bananas except Musa maclayi and Musa schizocarpa.

Figure 8. 95\% confidence intervals for mean long dimensions of leaf phytoliths differentiating Ensete glaucum from four other bananas.

some diploid cultivars commonly produce seed - cannot be differentiated. In these circumstances it is only the presence of Musaceae species outside their natural ranges that might imply human transmission and confirm evidence for cultivation.

Where seed is absent from archaeobotanical assemblages, the problem of identification becomes more difficult and is reliant on differentiation of the distinctive volcaniform morphotypes. From the results of several studies (Lentfer et al. 2003b, Mbida et al. 2001) it is well-established that Ensete species can be readily differentiated from wild and domesticated diploid and triploid Musa bananas by morphotypic and morphometric means. However, differentiation between Musa species is more
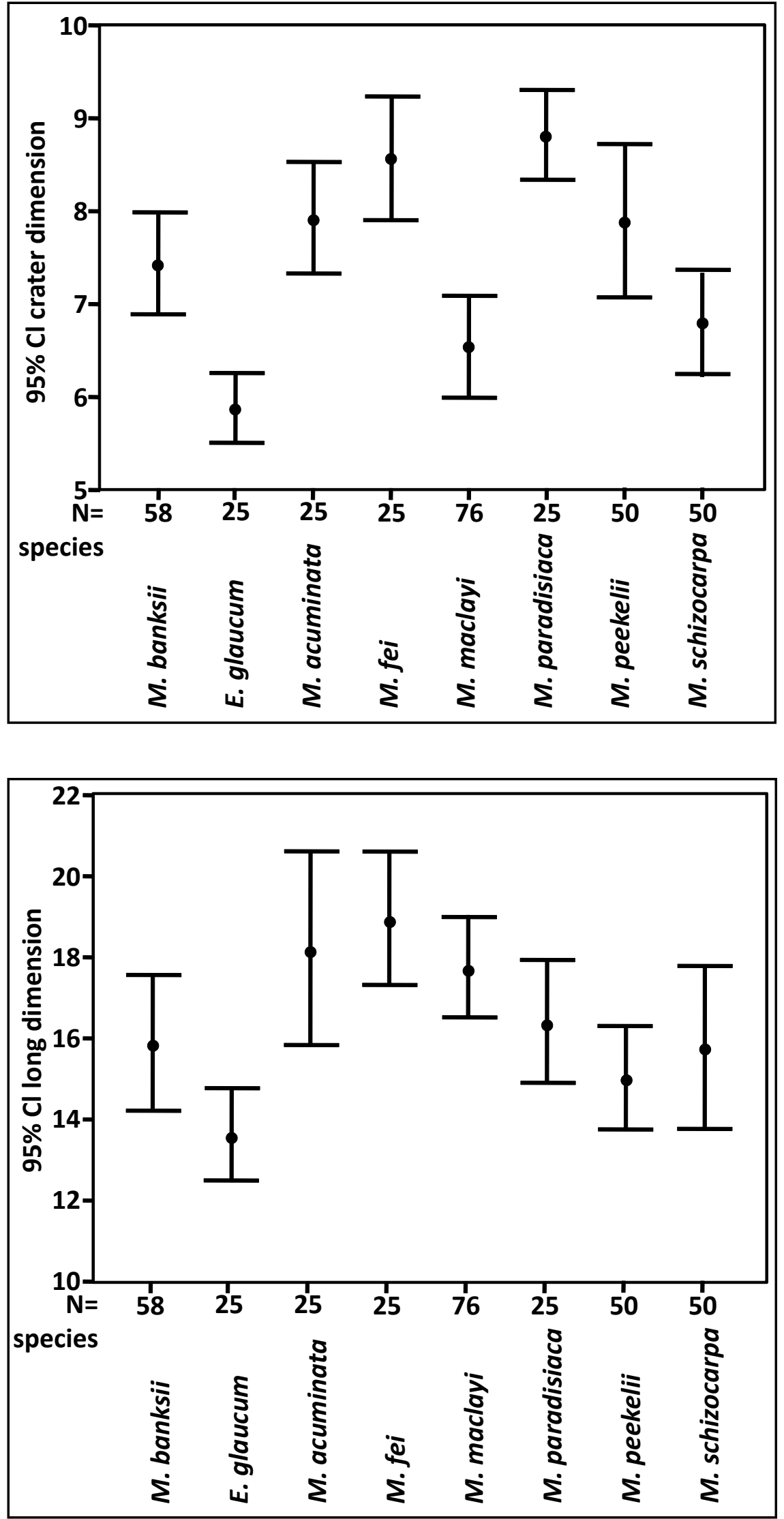


\section{Lentfer - Tracing Domestication and Cultivation of Bananas from Phytoliths: 257 An update from Papua New Guinea}

complex and would be reliant on a large sample size for any given archaeobotanical assemblage. Since triploid banana phytoliths are generally larger than diploids (Vrydaghs et al. 2009), there could be scope for differentiating domesticated triploid populations from wild and cultivated diploid populations by simply measuring crater widths of archaeological assemblages. Therefore, there is potential for tracking banana introductions and domestication. Nevertheless, results show that Eumusa and Australimusa section bananas cannot be differentiated at a general level and this is problematic in regions where bananas from both sections occur, either wild or cultivated. Indications are, however, that some species and/or cultivars within sections can be differentiated. Most importantly, wild and domesticated Eumusa bananas, M. acuminata $(\mathrm{AA})$ and $M$. balbisiana (BB), can be differentiated and there might be potential for tracking the introduction of Musa acuminata bananas in mainland Southeast Asia west

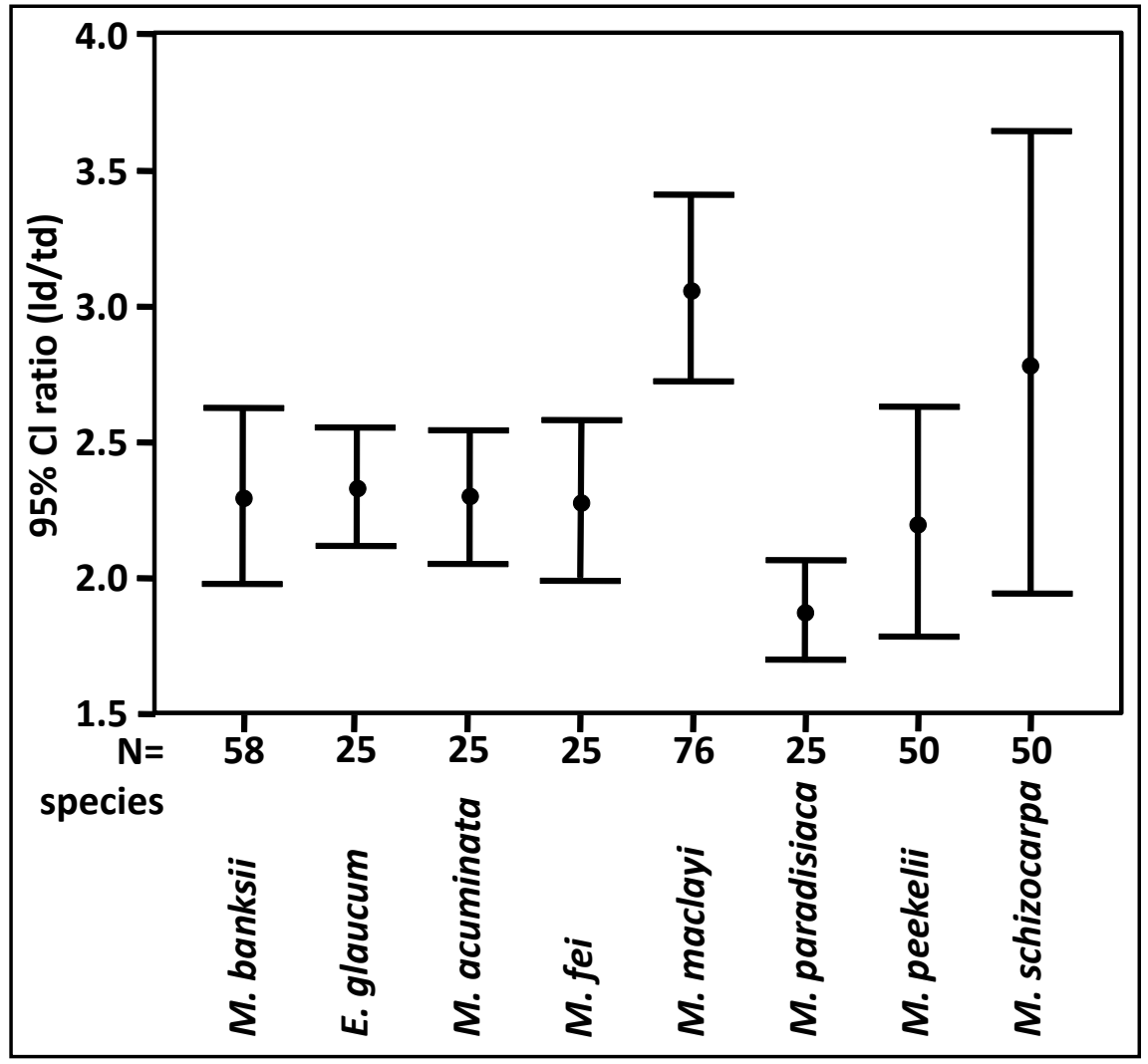

Figure 9. 95\% confidence intervals for mean long dimension (ld)/crater width (td) ratios of leaf phytoliths differentiating Musa maclayi from all other bananas except Musa schizocarpa. of the Philippines where $M$. balbisiana dominates native banana populations. A similar potential for discrimination is indicated for Australimusa; M. maclayi and $M$. peekelii could not only be differentiated from each other but also from the Australimusa domesticate Fe`i. Therefore, there may be good potential for tracking Australimusa banana dispersals and patterns of domestication for the near Oceania region, east of Papua New Guinea, where Australimusa bananas have dominated wild and cultivated populations.

\section{The 'New Guinea Banana Project'}

Good potential for differentiating between banana phytoliths is indicated from morphometric and morphotypic analyses. Nevertheless, preliminary studies point to a wide variation of morphotypes and additional study of a larger sample incorporating additional species and cultivars is required to determine the extent of this variation and further explore the potential for a more definitive set of criteria for differentiation. The 'New Guinea Banana Project' commenced in 2002 with collection of more than 100 wild and cultivated bananas from mainland Papua New Guinea, New Britain and New Ireland (Table 3). Volcaniform leaf phytoliths from 58 accessions were selected for a more rigorous analysis (Table 4) than previously under-

taken, describing more morphological features (Table 5). Digital images and measurements and morphometric details from 50 phytoliths per accession have been recorded and saved on a readily accessible database. This in itself is useful for identification of morphotypes during routine analysis. Statistical analyses have yet to be completed. Firstly, data will be lumped according to the same criteria as Ball et al. (2006) and examined using the same statistical procedure as Ball et al. (2006) and Vrydaghs et al. (2009) for direct comparison. Subsequently, the analysis will be re-run to incorporate the full set of morphotypic attributes and morphometries.

\section{Conclusions}

Recent research (Denham et al. 2003, Lebot 1999, Perrier et al. 2009) shows that the New Guinea region has played a key role in the development of the domesticated banana, and complex origins and multiple dispersals for banana cultivars within the southeast Asian/Pacific region are indicated (Kennedy 2008). Phytoliths hold the key to tracing the history of banana cultivation and domestication in the archaeobotanical record, and morphometric and morphotypic analyses show good potential for differentiation of phytoliths. Seed phytoliths can be readily dif- 


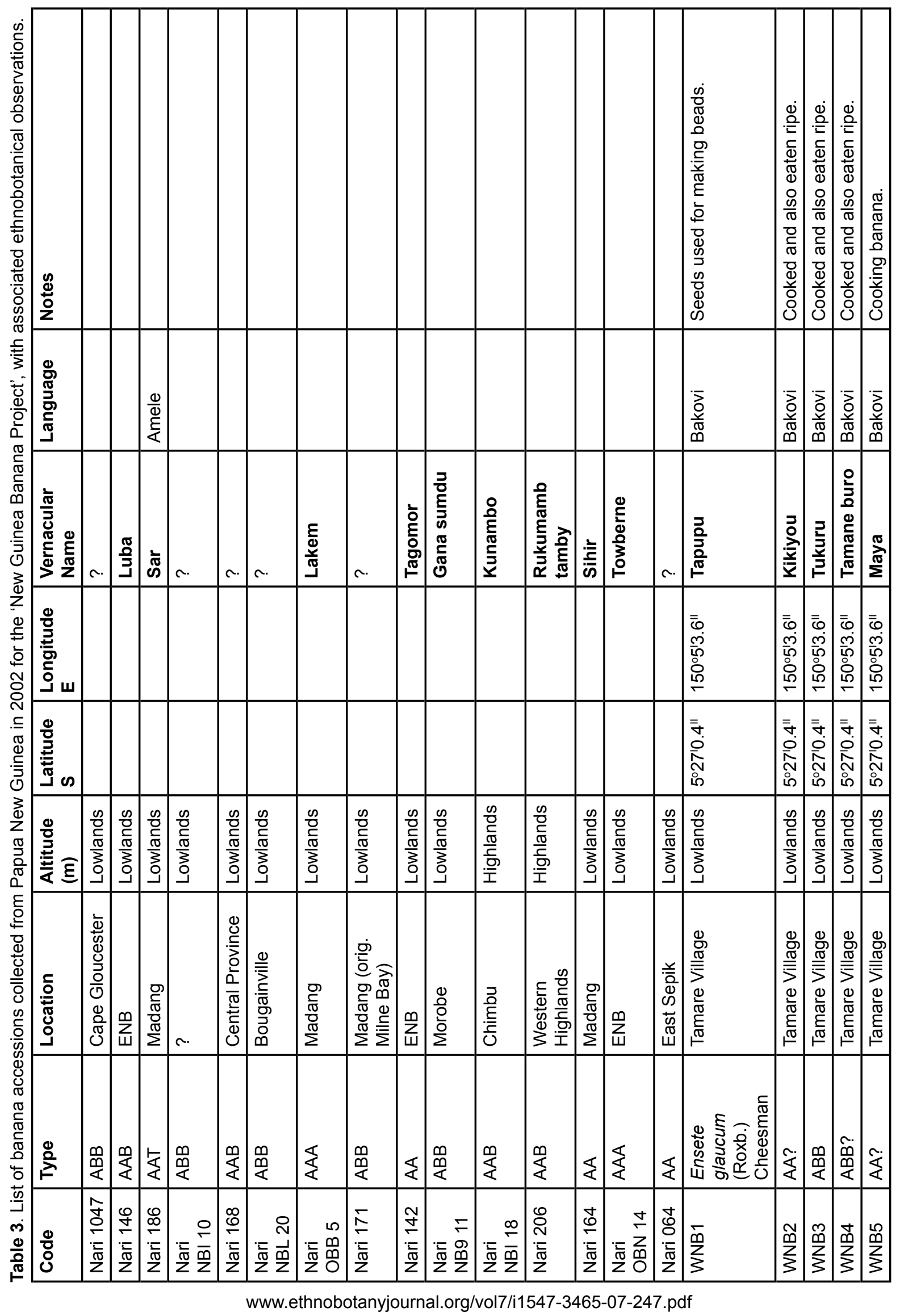




\begin{tabular}{|c|c|c|c|c|c|c|c|c|c|c|c|c|}
\hline $\begin{array}{l}\text { d) } \\
\text { t0 } \\
\text { z }\end{array}$ & 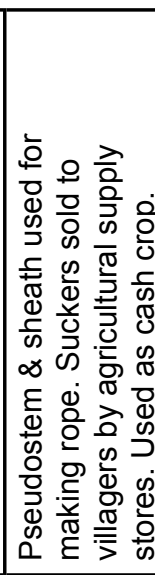 & 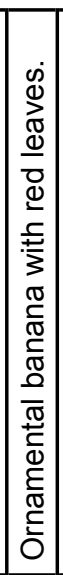 & 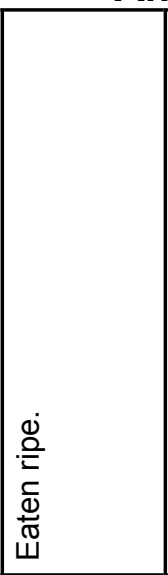 & 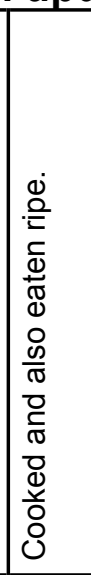 & 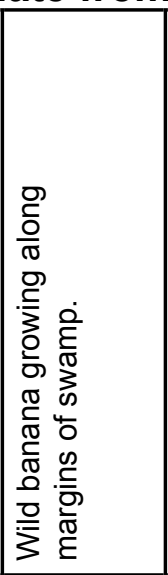 & 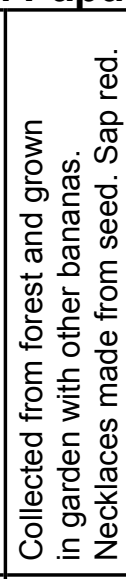 & 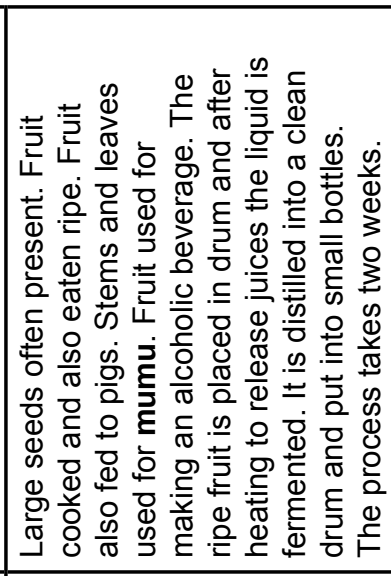 & 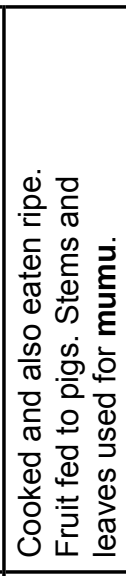 & 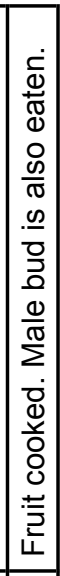 & 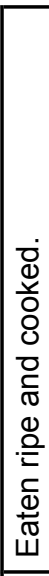 & \begin{tabular}{|c|} 
\\
\\
\\
\end{tabular} & 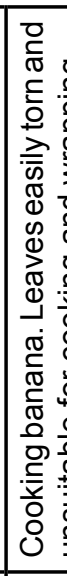 \\
\hline 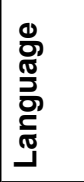 & $\frac{. \frac{5}{c 0}}{\frac{0}{0}}$ & $\begin{array}{l}\bar{z} \\
\overline{0} \\
\overline{\mathbb{N}} \\
\bar{\infty}\end{array}$ & $\begin{array}{l}\bar{z} \\
\frac{\partial}{\tilde{\sigma}} \\
\tilde{\Phi}\end{array}$ & 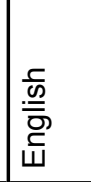 & $\frac{\frac{5}{00}}{\frac{0}{2}}$ & 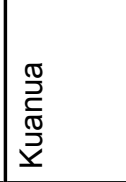 & 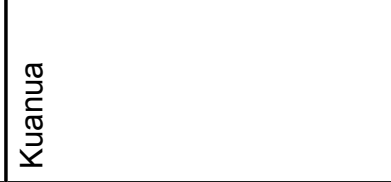 & 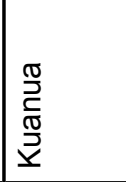 & $\begin{array}{l}\frac{0}{2} \\
\frac{\vec{T}}{\tilde{T}} \\
\frac{\vec{T}}{2}\end{array}$ & $\begin{array}{l}\frac{\sigma}{2} \\
\frac{\tilde{T}}{\tilde{\nu}} \\
\underline{\underline{\nu}}\end{array}$ & \begin{tabular}{|l|}
$\frac{\sigma}{2}$ \\
$\frac{\tilde{T}}{\tilde{T}}$ \\
$\frac{\hat{2}}{2}$
\end{tabular} & 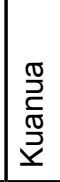 \\
\hline 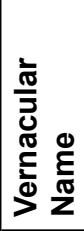 & 旅 & 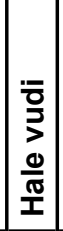 & 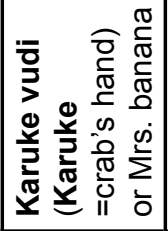 & 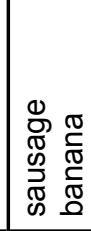 & 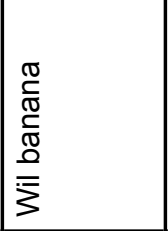 & 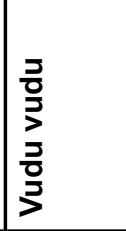 & 疍 & 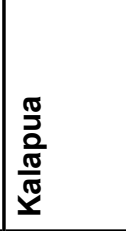 & 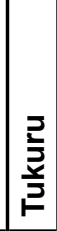 & 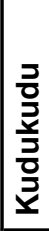 & 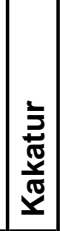 & 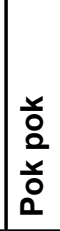 \\
\hline 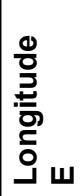 & $\begin{array}{l}\overline{0} \\
0 \\
0 \\
10 \\
0 \\
0 \\
10 \\
10\end{array}$ & 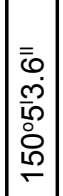 & 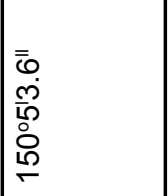 & 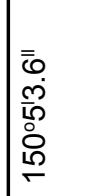 & 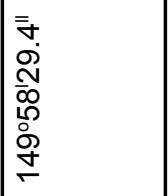 & 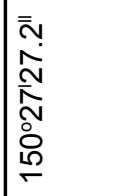 & 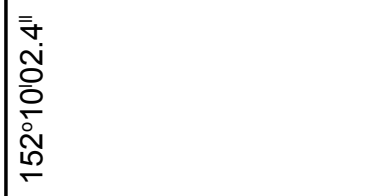 & 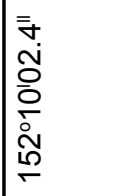 & 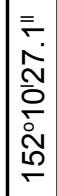 & 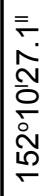 & $\begin{array}{l} \\
0 \\
0 \\
0 \\
0 \\
0 \\
0 \\
0 \\
i \\
0 \\
\end{array}$ & 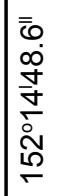 \\
\hline 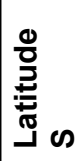 & 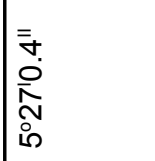 & 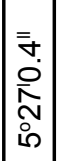 & 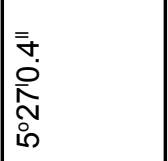 & 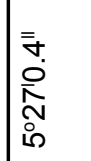 & 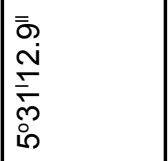 & 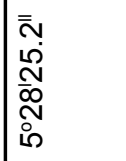 & 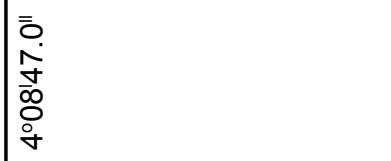 & 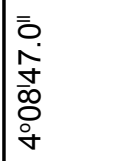 & \begin{tabular}{|l|}
$\bar{\sigma}$ \\
$\infty$ \\
$\infty$ \\
10 \\
$\infty$ \\
0 \\
$\dot{0}$ \\
$\dot{\gamma}$
\end{tabular} & 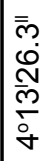 & $\begin{array}{l}\bar{\sigma} \\
\infty \\
\infty \\
0 \\
\infty \\
0 \\
0 \\
\dot{+}\end{array}$ & 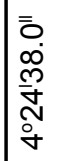 \\
\hline 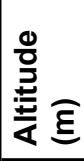 & \begin{tabular}{|l}
0 \\
0 \\
$\frac{0}{\pi}$ \\
$\frac{\pi}{3}$ \\
0 \\
0
\end{tabular} & $\begin{array}{l}0 \\
0 \\
\frac{2}{\pi} \\
\frac{\pi}{3} \\
0 \\
-1\end{array}$ & \begin{tabular}{|l}
0 \\
0 \\
0 \\
$\frac{0}{\pi}$ \\
$\frac{\pi}{3}$ \\
0 \\
\end{tabular} & \begin{tabular}{|l}
0 \\
0 \\
0 \\
$\frac{0}{\pi}$ \\
$\frac{\pi}{3}$ \\
0 \\
\end{tabular} & $\begin{array}{l}0 \\
0 \\
0 \\
\frac{1}{\pi} \\
3 \\
0 \\
\end{array}$ & \begin{tabular}{|l}
0 \\
0 \\
$\frac{0}{0}$ \\
$\frac{\pi}{3}$ \\
0 \\
0 \\
\end{tabular} & \begin{tabular}{|l}
0 \\
0 \\
$\frac{1}{0}$ \\
$\frac{\pi}{\pi}$ \\
3 \\
0 \\
\end{tabular} & \begin{tabular}{|l}
0 \\
0 \\
$\frac{1}{0}$ \\
$\frac{\pi}{3}$ \\
3 \\
0 \\
\end{tabular} & \begin{tabular}{|l|}
0 \\
0 \\
$\frac{0}{\pi}$ \\
$\frac{1}{3}$ \\
0 \\
0
\end{tabular} & $\begin{array}{l}0 \\
0 \\
\frac{1}{0} \\
\frac{\pi}{3} \\
0 \\
\end{array}$ & $\begin{array}{l}0 \\
0 \\
\frac{0}{0} \\
\frac{\pi}{3} \\
0 \\
\end{array}$ & $\begin{array}{l}0 \\
0 \\
\frac{0}{C} \\
\frac{\pi}{3} \\
0 \\
\end{array}$ \\
\hline 음 & 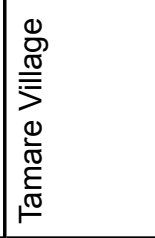 & 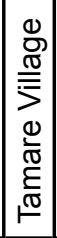 & 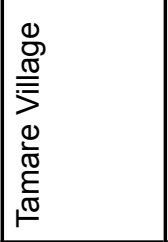 & 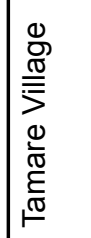 & 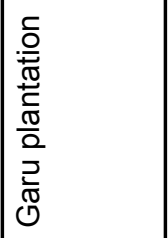 & 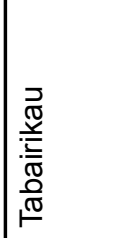 & 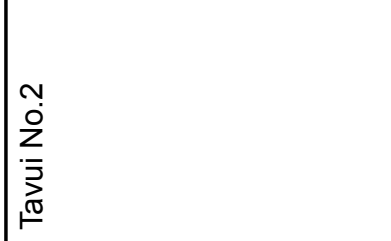 & 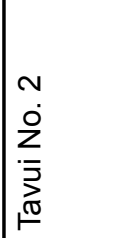 & 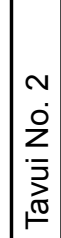 & 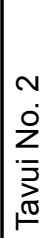 & $\begin{array}{l}\frac{\pi}{2} \\
\frac{\sigma}{5} \\
\frac{\pi}{0} \\
\frac{\pi}{20}\end{array}$ & \begin{tabular}{|l}
$\frac{\pi}{8}$ \\
0 \\
$\frac{\pi}{0}$ \\
0 \\
0
\end{tabular} \\
\hline$\sum_{2}^{\Perp}$ & 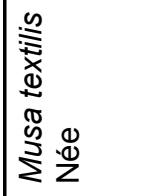 & $\sim$ & 这 & 这 & 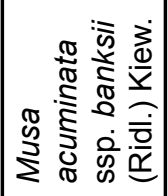 & 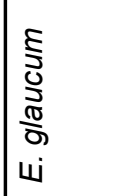 & 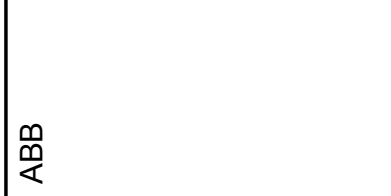 & 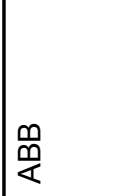 & 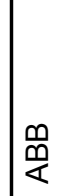 & $\sim$ & r. & . \\
\hline $\begin{array}{l}0 \\
\\
0\end{array}$ & 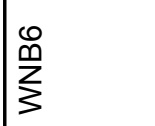 & $\frac{\hat{n}}{z}$ & 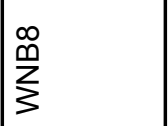 & 呑 & $\frac{0}{\frac{0}{m}}$ & 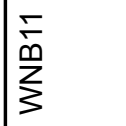 & 㐫 & 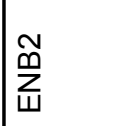 & $\sum_{\text {u }}^{\tilde{m}}$ & 岕 & 邑 & $\mid \begin{array}{l}\stackrel{\ominus}{2} \\
\stackrel{M}{Z} \\
\end{array}$ \\
\hline
\end{tabular}




\begin{tabular}{|c|c|c|c|c|c|c|c|c|c|c|c|c|c|c|c|c|c|c|c|}
\hline 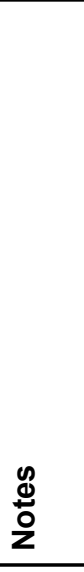 & 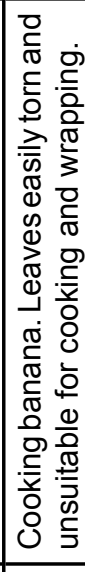 & 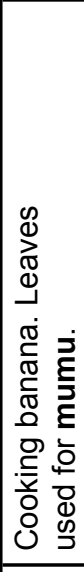 & 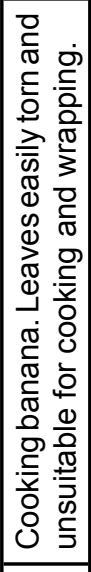 & 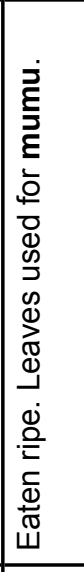 & 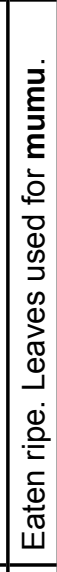 & 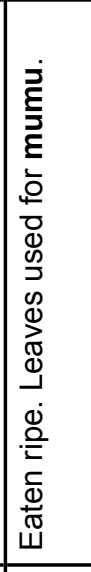 & 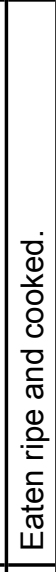 & 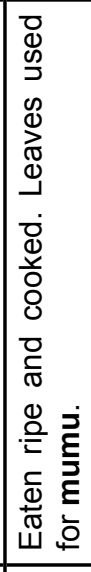 & 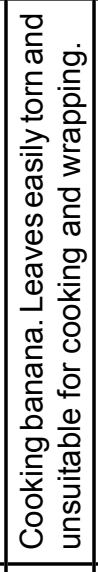 & 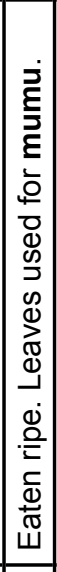 & 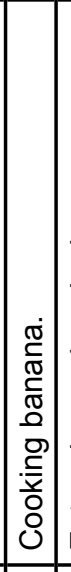 & 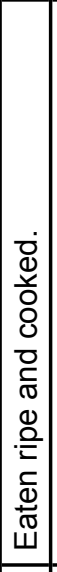 & & 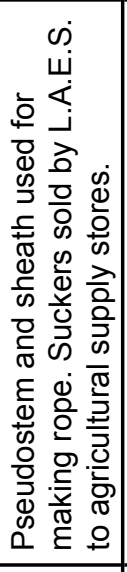 & 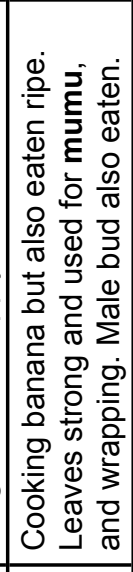 & 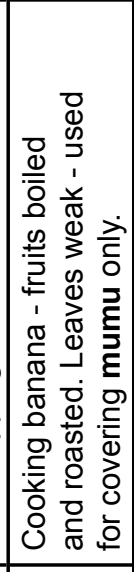 & 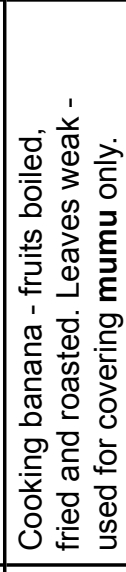 & 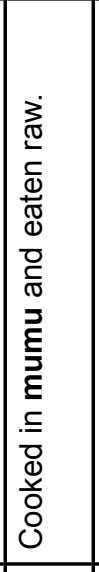 & 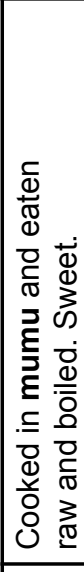 \\
\hline 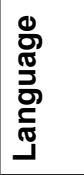 & 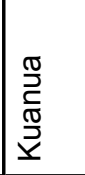 & 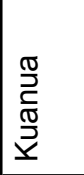 & 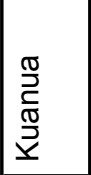 & 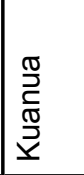 & 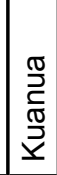 & 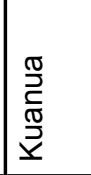 & 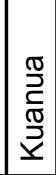 & 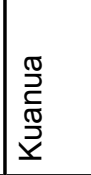 & 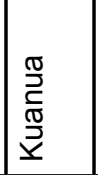 & 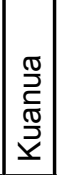 & $\mid \begin{array}{l}\frac{\pi}{0} \\
\frac{2}{T} \\
\frac{\sigma}{2} \\
\underline{2}\end{array}$ & 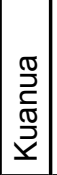 & & & 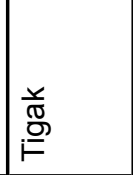 & 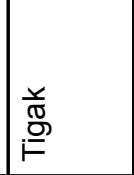 & 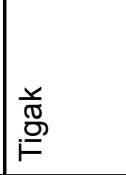 & $\frac{\frac{5}{60}}{\frac{0}{2}}$ & 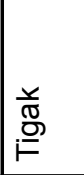 \\
\hline 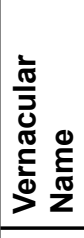 & 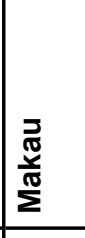 & 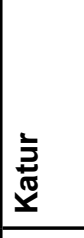 & $\frac{z}{\Delta}$ & 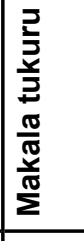 & 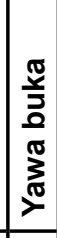 & 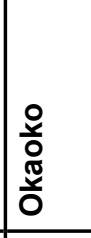 & \begin{tabular}{|l|}
0 \\
0 \\
3 \\
11 \\
0 \\
0 \\
3 \\
\\
\end{tabular} & 일 & \begin{tabular}{|l}
$\frac{E}{3}$ \\
$\frac{3}{5}$ \\
$\mathcal{0}$
\end{tabular} & 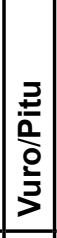 & 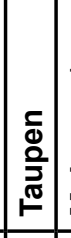 & 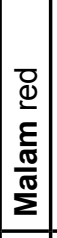 & & & 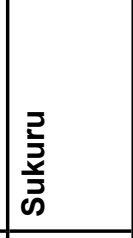 & 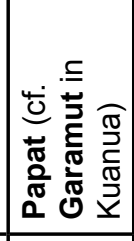 & 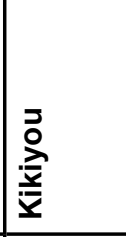 & 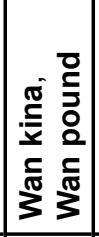 & ס̊ \\
\hline 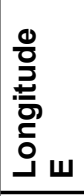 & 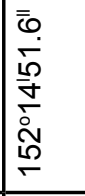 & 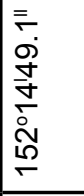 & 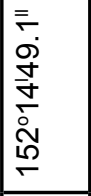 & 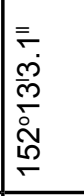 & 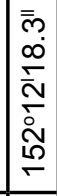 & 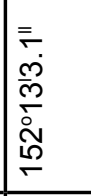 & 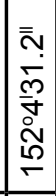 & 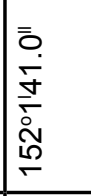 & 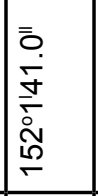 & 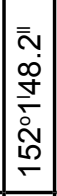 & 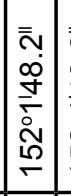 & 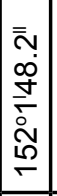 & 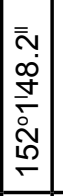 & 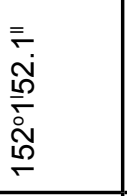 & 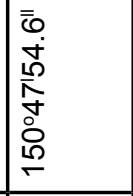 & 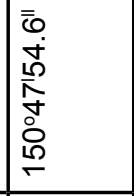 & 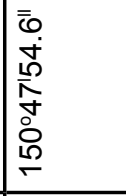 & 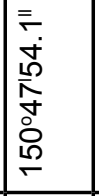 & 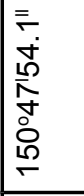 \\
\hline 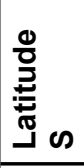 & 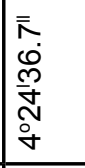 & 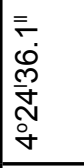 & 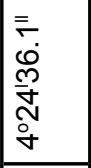 & 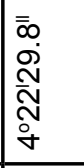 & 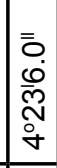 & 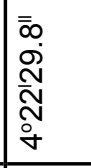 & 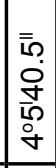 & 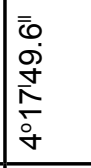 & 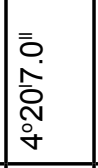 & 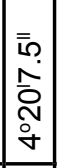 & 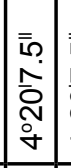 & 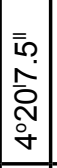 & 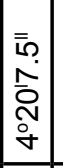 & 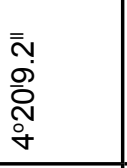 & \begin{tabular}{|l}
$\overline{\tilde{m}}$ \\
$\tilde{w}$ \\
$\tilde{m}$ \\
$\stackrel{\tilde{N}}{ }$
\end{tabular} & 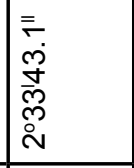 & 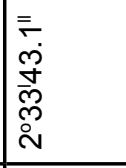 & 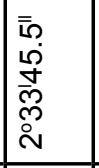 & 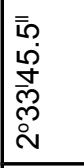 \\
\hline 总 & \begin{tabular}{|l}
0 \\
0 \\
$\frac{1}{0}$ \\
$\frac{0}{3}$ \\
0 \\
\end{tabular} & $\begin{array}{l}00 \\
0 \\
\frac{0}{0} \\
\frac{\pi}{3} \\
0 \\
\end{array}$ & $\begin{array}{l}0 \\
0 \\
\frac{1}{0} \\
\frac{\pi}{3} \\
0 \\
\end{array}$ & \begin{tabular}{|l}
$\frac{0}{0}$ \\
$\frac{0}{6}$ \\
$\frac{\pi}{3}$ \\
3 \\
\\
\end{tabular} & $\begin{array}{l}0 \\
0 \\
\frac{0}{0} \\
\frac{0}{3} \\
0 \\
\end{array}$ & \begin{tabular}{|l}
$\frac{0}{0}$ \\
0 \\
$\frac{\pi}{3}$ \\
$\frac{3}{3}$ \\
0 \\
\end{tabular} & \begin{tabular}{|l|}
0 \\
0 \\
$\frac{1}{0}$ \\
$\frac{0}{3}$ \\
0 \\
\\
\end{tabular} & \begin{tabular}{|l}
$\frac{0}{0}$ \\
$\frac{0}{0}$ \\
$\frac{\pi}{3}$ \\
3 \\
0 \\
\end{tabular} & \begin{tabular}{|l}
0 \\
0 \\
$\frac{0}{0}$ \\
$\frac{\pi}{3}$ \\
0 \\
\end{tabular} & \begin{tabular}{|l|}
0 \\
0 \\
$\frac{0}{0}$ \\
$\frac{0}{3}$ \\
0 \\
\\
\end{tabular} & \begin{tabular}{|c|}
0 \\
0 \\
$\frac{0}{0}$ \\
$\frac{0}{3}$ \\
0 \\
0 \\
\end{tabular} & \begin{tabular}{|}
0 \\
$\frac{1}{2}$ \\
$\frac{\pi}{\pi}$ \\
$\frac{\pi}{3}$ \\
0 \\
\end{tabular} & \begin{tabular}{|l|}
0 \\
0 \\
$\frac{1}{0}$ \\
3 \\
0 \\
\end{tabular} & $\begin{array}{l}\frac{0}{0} \\
\frac{0}{0} \\
\frac{\pi}{3} \\
0 \\
\end{array}$ & \begin{tabular}{|l|}
0 \\
0 \\
$\frac{1}{0}$ \\
$\frac{\pi}{3}$ \\
0 \\
\end{tabular} & \begin{tabular}{|l}
0 \\
$\frac{0}{0}$ \\
$\frac{1}{\pi}$ \\
$\frac{\pi}{3}$ \\
0 \\
\end{tabular} & $\begin{array}{l}\frac{0}{0} \\
\frac{1}{0} \\
\frac{\pi}{3} \\
0 \\
\end{array}$ & \begin{tabular}{|l|}
0 \\
0 \\
$\frac{1}{0}$ \\
$\frac{\pi}{3}$ \\
0 \\
\end{tabular} & \begin{tabular}{|l}
$\frac{0}{0}$ \\
$\frac{0}{0}$ \\
$\frac{\pi}{3}$ \\
0 \\
\\
\end{tabular} \\
\hline 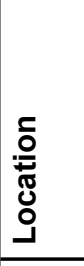 & $\begin{array}{l}\frac{\pi}{10} \\
0 \\
\frac{\pi}{10} \\
0\end{array}$ & $\begin{array}{l}\frac{\pi}{0} \\
0 \\
\frac{\pi}{0} \\
0 \\
\end{array}$ & $\begin{array}{l}\frac{\pi}{10} \\
0 \\
\frac{\pi}{0} \\
0 \\
\end{array}$ & 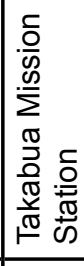 & 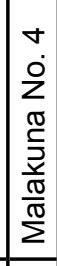 & 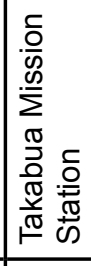 & \begin{tabular}{|l} 
\\
\\
$\frac{0}{0}$ \\
$\frac{\pi}{\alpha}$ \\
$\square$
\end{tabular} & 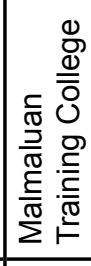 & 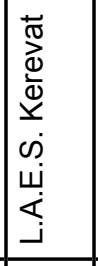 & 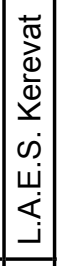 & 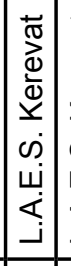 & 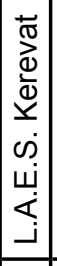 & 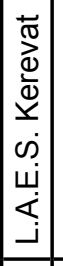 & 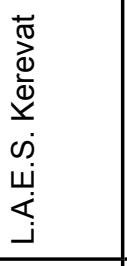 & 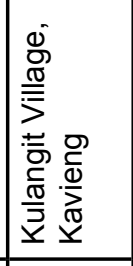 & 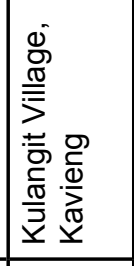 & 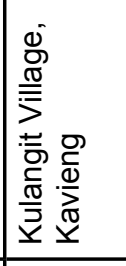 & 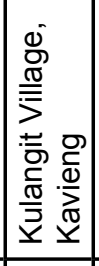 & 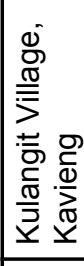 \\
\hline$\stackrel{\Perp}{\circledR}$ & $\curvearrowright$ & $\frac{8}{4}$ & 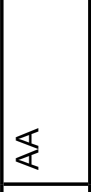 & $\begin{array}{l}\frac{m}{q} \\
\frac{m}{<}\end{array}$ & 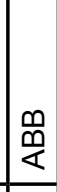 & $\ddot{m}$ & \begin{tabular}{|l|}
0 \\
4 \\
\end{tabular} & 选 & $\$$ & 迨 & 离 & $\frac{1}{4}$ & 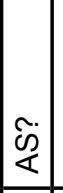 & 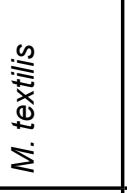 & 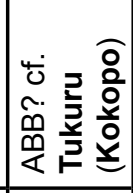 & $\frac{8}{8}$ & $\ll$ & 管 & \\
\hline $\begin{array}{l}0 \\
\text { ¿ } \\
0\end{array}$ & 全 & $\sum_{\text {离 }}^{\infty}$ & 离 & 品 & $\underset{\dot{m}}{\stackrel{\Sigma}{\mathbf{m}}}$ & $\underset{\text { W }}{\stackrel{N}{\mathbf{M}}}$ & $\frac{m}{\bar{m}}$ & $\underset{\Psi}{\stackrel{v}{M}}$ & $\frac{10}{\sum_{w}^{2}}$ & $\begin{array}{l}\frac{0}{m} \\
\sum_{\Psi} \\
\end{array}$ & \begin{tabular}{|l} 
\\
$\bar{m}$ \\
$\bar{\Psi}$ \\
\end{tabular} & $\frac{\infty}{m}$ & \begin{tabular}{|l|} 
\\
$\underline{m}$ \\
\end{tabular} & 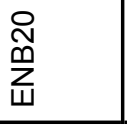 & $\overline{\bar{z}}$ & $\stackrel{N}{Z}$ & $\frac{m}{z}$ & $\frac{ \pm}{z}$ & $\frac{10}{z}$ \\
\hline
\end{tabular}


Lentfer - Tracing Domestication and Cultivation of Bananas from Phytoliths: 261 An update from Papua New Guinea

\begin{tabular}{|c|c|c|c|c|c|c|c|c|c|c|c|c|c|c|}
\hline 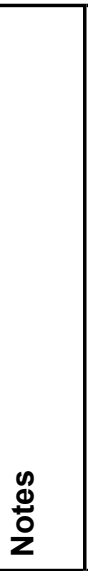 & 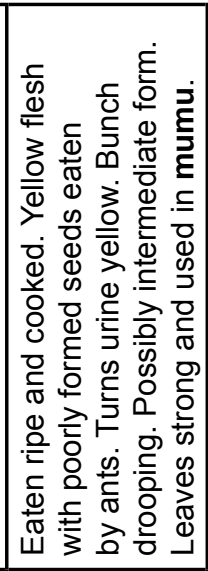 & 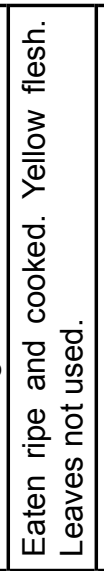 & 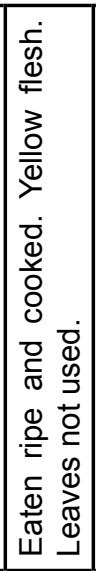 & 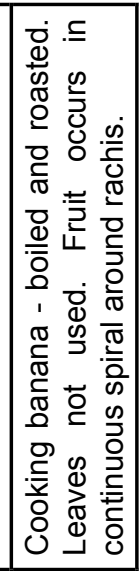 & 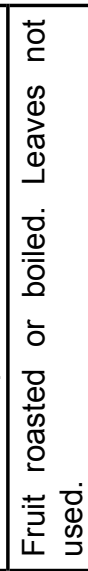 & 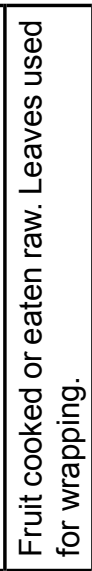 & 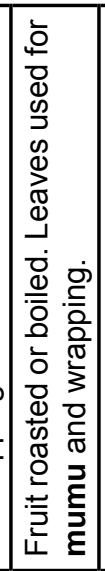 & 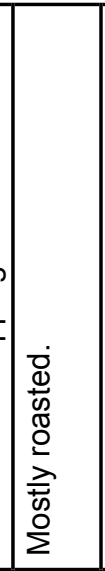 & 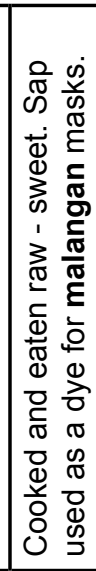 & 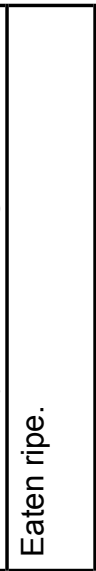 & 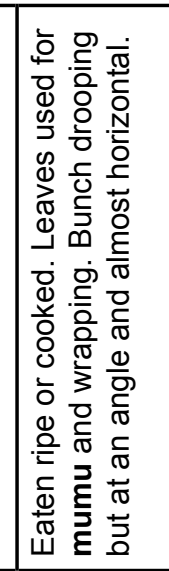 & 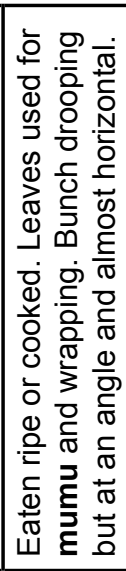 & 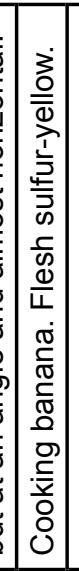 & 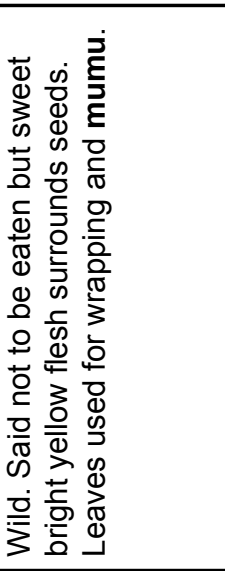 \\
\hline 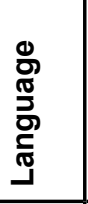 & 总 & 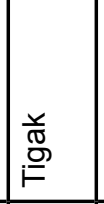 & 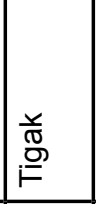 & 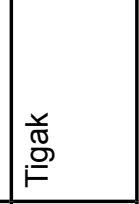 & 莺 & 兽 & 龸 & 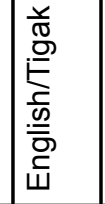 & $\mid \frac{\underline{\underline{\underline{I}}}}{\sum^{\bar{\omega}}}$ & \begin{tabular}{|l}
$\frac{x}{\pi}$ \\
$\frac{\pi}{0}$ \\
$\frac{\pi}{\pi}$ \\
$\Sigma$
\end{tabular} & 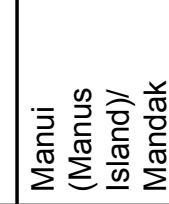 & 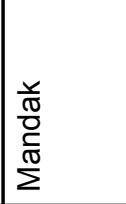 & $\mid$\begin{tabular}{|c|}
$\frac{2}{\pi}$ \\
$\frac{10}{0}$ \\
$\frac{10}{20}$ \\
2
\end{tabular} & 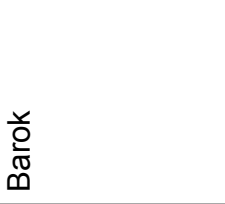 \\
\hline 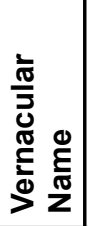 & 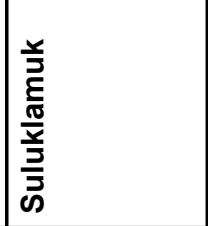 & 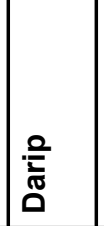 & 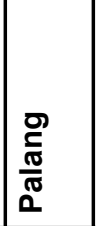 & 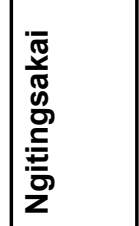 & 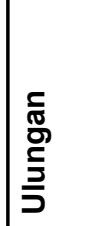 & 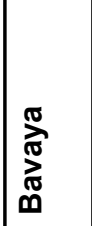 & 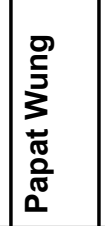 & 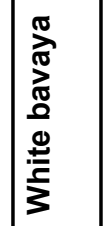 & 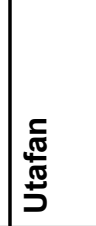 & 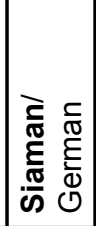 & 竞 & $\mid$ & 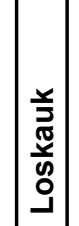 & ") \\
\hline 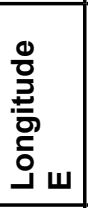 & 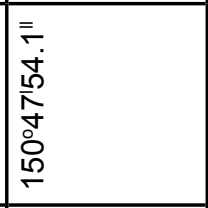 & 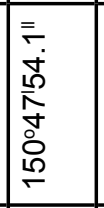 & 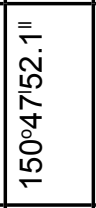 & 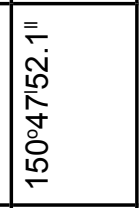 & 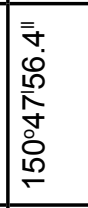 & 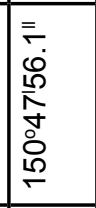 & 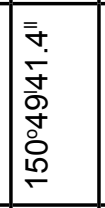 & 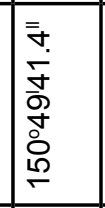 & 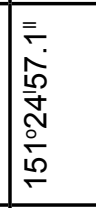 & 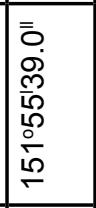 & 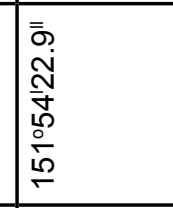 & 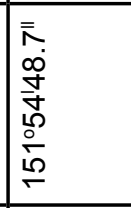 & 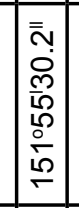 & 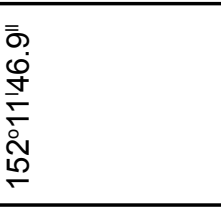 \\
\hline 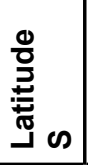 & 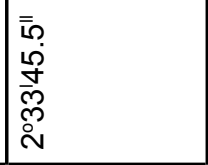 & $\mid$ & 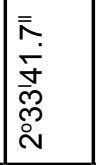 & 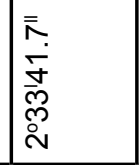 & 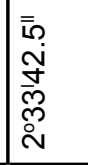 & 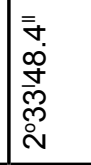 & 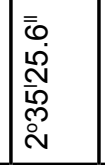 & 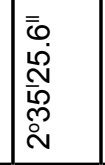 & 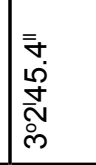 & 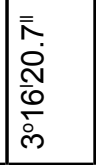 & 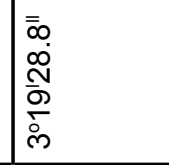 & 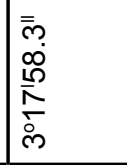 & $\mid$\begin{tabular}{|c|}
$=$ \\
0 \\
0 \\
0 \\
0 \\
$\dot{m}$ \\
\end{tabular} & 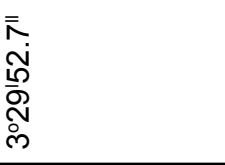 \\
\hline 这 & 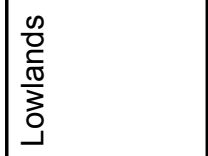 & 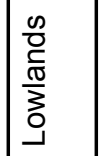 & \begin{tabular}{|l}
$\frac{0}{0}$ \\
$\frac{1}{5}$ \\
$\frac{\pi}{3}$ \\
3 \\
0
\end{tabular} & \begin{tabular}{|l}
0 \\
0 \\
0 \\
$\frac{\pi}{3}$ \\
3 \\
0 \\
9
\end{tabular} & $\begin{array}{l}\frac{0}{2} \\
0 \\
\frac{0}{3} \\
0 \\
0\end{array}$ & $\begin{array}{l}\frac{0}{0} \\
0 \\
\frac{\pi}{3} \\
3 \\
0 \\
9\end{array}$ & \begin{tabular}{|l}
0 \\
0 \\
0 \\
$\frac{0}{3}$ \\
0 \\
0
\end{tabular} & \begin{tabular}{|l}
$\frac{0}{0}$ \\
0 \\
$\frac{\pi}{3}$ \\
3 \\
0 \\
0
\end{tabular} & \begin{tabular}{|l}
$\frac{1}{0}$ \\
$\frac{0}{5}$ \\
$\frac{\pi}{3}$ \\
3 \\
0 \\
9
\end{tabular} & $\frac{\varepsilon}{\grave{g}}$ & $\frac{\bar{\delta}}{\mathrm{g}}$ & $\begin{array}{l}E \\
\bar{O} \\
\bar{v} \\
\mathrm{v}\end{array}$ & $\mid \begin{array}{l}E \\
\bar{\partial} \\
\grave{v}\end{array}$ & $\begin{array}{l}\varepsilon \\
\grave{Q} \\
\grave{v}\end{array}$ \\
\hline 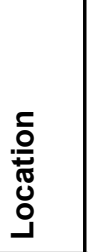 & 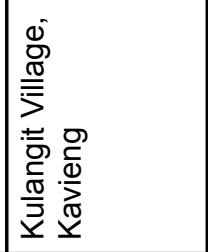 & 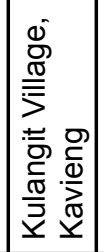 & 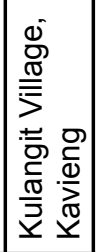 & 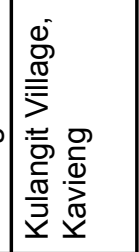 & 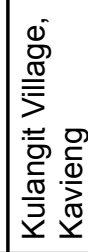 & 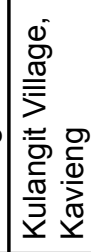 & 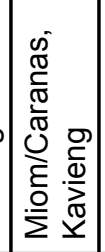 & 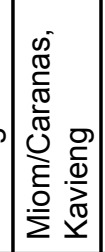 & 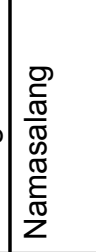 & $\frac{\vec{\omega}}{\Phi}$ & 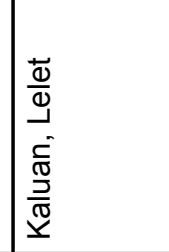 & 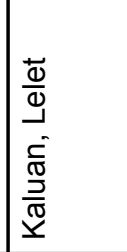 & 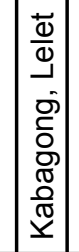 & 로 \\
\hline$\stackrel{\otimes}{\stackrel{2}{2}}$ & 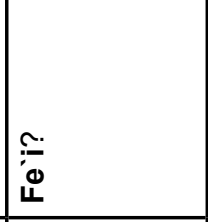 & م. & $\frac{\$}{4}$ & $\varangle$ & $\varangle$ & $\frac{m}{\frac{m}{\alpha}}$ & $\varangle$ & $\frac{m}{k}$ & 䘡 & r. & 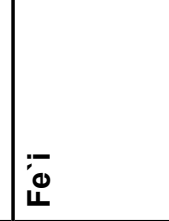 & 离 & \begin{tabular}{|c|} 
\\
$:-$ \\
0 \\
4
\end{tabular} & 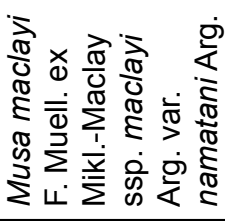 \\
\hline ¿ّ̊ & $\frac{\varphi}{z}$ & $\hat{\bar{z}}$ & $\frac{\infty}{z}$ & $\frac{9}{z}$ & $\mid \frac{}{z}$ & $\frac{\bar{E}}{\bar{z}}$ & $\frac{N}{\bar{z}}$ & $\frac{m}{z}$ & $\frac{\mathbb{Z}}{\bar{z}}$ & $\mid \frac{10}{z}$ & $\frac{0}{\bar{z}}$ & $\mid \frac{\hat{L}}{z}$ & $\left|\frac{\infty}{\bar{z}}\right|$ & $\frac{O}{\bar{z}}$ \\
\hline
\end{tabular}




\begin{tabular}{|c|c|c|c|c|c|c|c|c|c|c|}
\hline $\begin{array}{l}\stackrel{y}{ \pm} \\
\stackrel{0}{z}\end{array}$ & 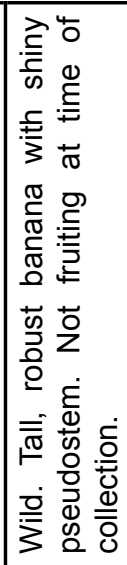 & 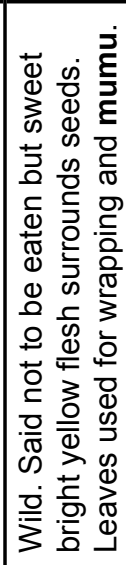 & 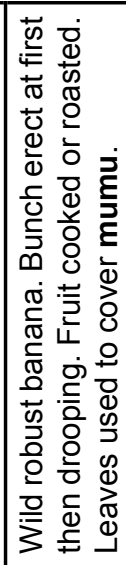 & 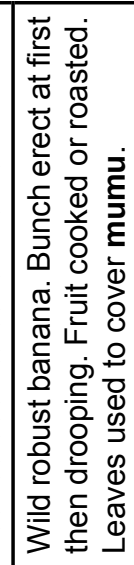 & 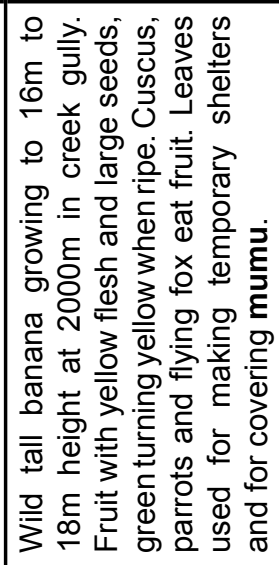 & 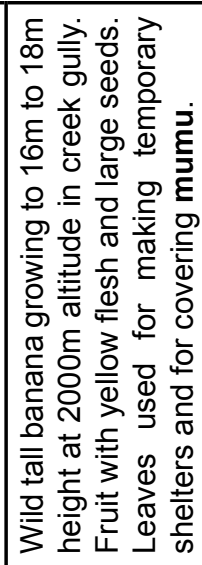 & \begin{tabular}{|l|}
0 \\
0 \\
0 \\
$\frac{8}{\pi}$ \\
0 \\
$\frac{9}{2}$
\end{tabular} & 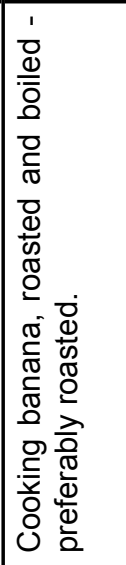 & 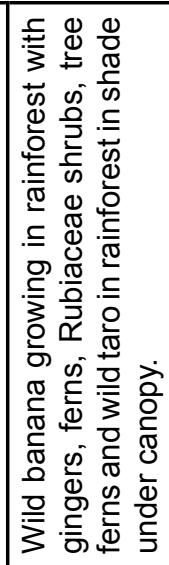 & 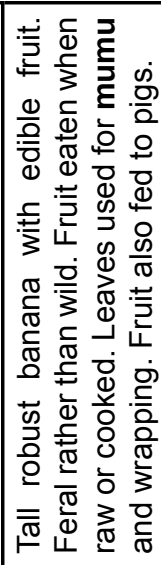 \\
\hline $\begin{array}{l}0 \\
\frac{0}{0} \\
5 \\
\frac{\pi}{\pi} \\
\text { J }\end{array}$ & \begin{tabular}{|l} 
\\
0 \\
$\stackrel{0}{\mathbb{N}}$ \\
$\infty$
\end{tabular} & 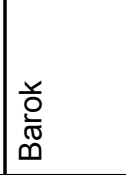 & 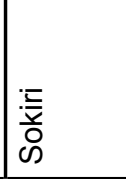 & $\begin{array}{l}\text { 咅 } \\
\text { ○ }\end{array}$ & 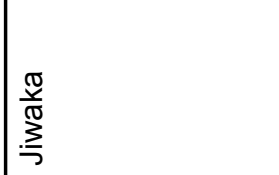 & 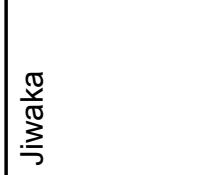 & $\begin{array}{l}\frac{\pi}{2} \\
\frac{2}{\pi} \\
\underline{\underline{n}}\end{array}$ & & r. & $\stackrel{\stackrel{\circ}{(}}{\sum}$ \\
\hline 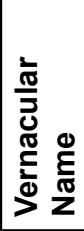 & & 票 & 焉 & 要 & 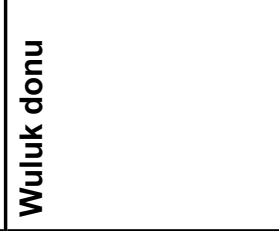 & \begin{tabular}{|l}
$\vec{z}$ \\
$\vdots$ \\
0 \\
0 \\
$\frac{1}{3}$ \\
$\frac{3}{3}$ \\
3
\end{tabular} & $\begin{array}{l}z \\
\frac{2}{2} \\
0 \\
\frac{y}{3} \\
\frac{y}{3} \\
3 \\
3\end{array}$ & 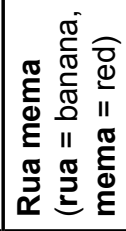 & . & 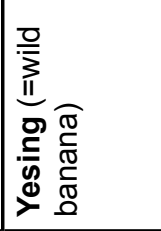 \\
\hline 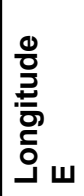 & 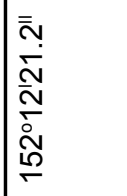 & 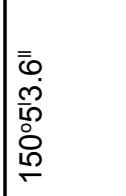 & 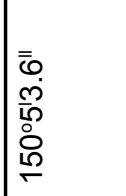 & 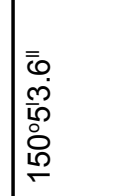 & 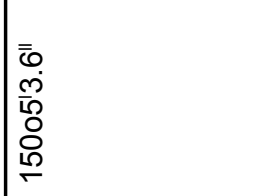 & 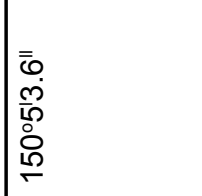 & $\begin{array}{l}\overline{0} \\
0 \\
0 \\
0 \\
0 \\
0 \\
10\end{array}$ & 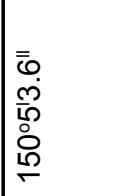 & $\begin{array}{l}\bar{\sigma} \\
0 \\
0 \\
0 \\
0 \\
10 \\
10\end{array}$ & 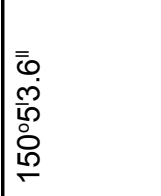 \\
\hline 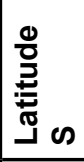 & 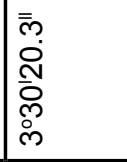 & 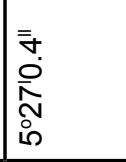 & 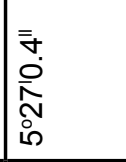 & 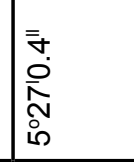 & 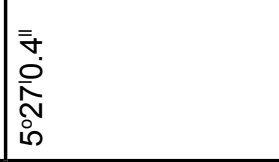 & 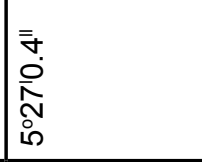 & 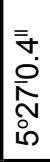 & 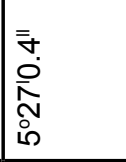 & 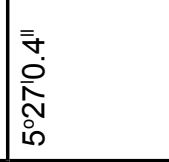 & 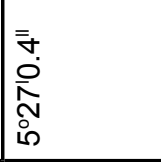 \\
\hline 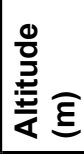 & $\mid \begin{array}{l}\varepsilon \\
\grave{O} \\
\bar{v}\end{array}$ & $\frac{\bar{\delta}}{\grave{v}}$ & $\frac{\bar{\delta}}{\grave{v}}$ & $\frac{\bar{\delta}}{\mathrm{O}}$ & E్ & E్ & हి & 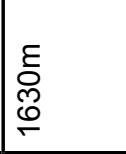 & 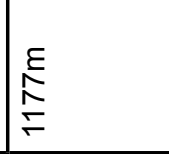 & $\begin{array}{l}\frac{0}{0} \\
\frac{0}{\pi} \\
\frac{\pi}{3} \\
0 \\
0\end{array}$ \\
\hline 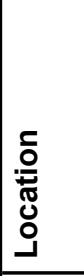 & 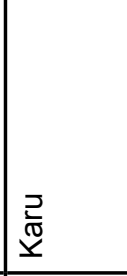 & \begin{tabular}{|l}
2 \\
$\stackrel{2}{\pi}$ \\
$\underline{x}$ \\
\end{tabular} & 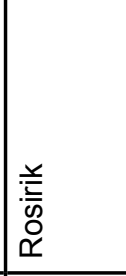 & 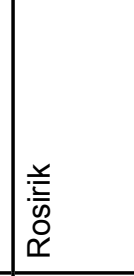 & 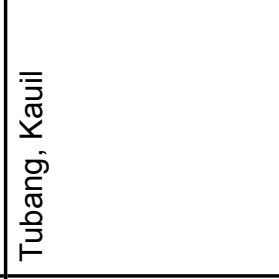 & 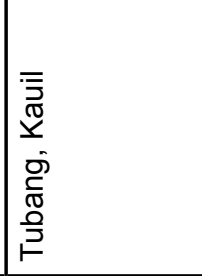 & 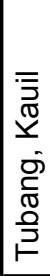 & 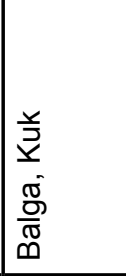 & $\begin{array}{l}8 \\
\stackrel{0}{N} \\
\bar{\Phi} \\
\stackrel{\bar{D}}{\bar{N}} \\
\infty\end{array}$ & 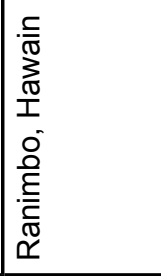 \\
\hline 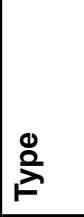 & 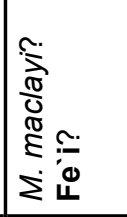 & 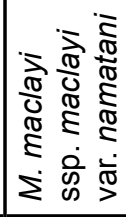 & 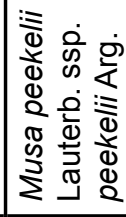 & 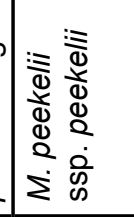 & 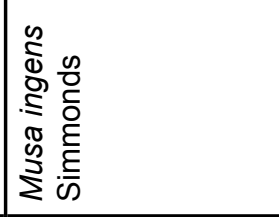 & 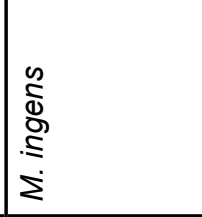 & 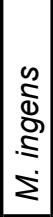 & :- & 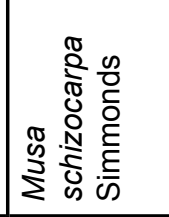 & 定 \\
\hline 苋 & $\frac{\stackrel{O}{z}}{z}$ & $\frac{\bar{N}}{\bar{z}}$ & $\frac{\mathbb{N}}{z}$ & $\stackrel{\stackrel{N}{z}}{z}$ & $\stackrel{\bar{Y}}{3}$ & $\frac{\stackrel{N}{Y}}{3}$ & $\frac{m}{M^{T}}$ & $\stackrel{+}{\stackrel{T}{3}}$ & $\frac{10}{1}$ & $\bar{c}$ \\
\hline
\end{tabular}


An update from Papua New Guinea

\begin{tabular}{|c|c|c|c|c|c|c|c|c|c|c|}
\hline 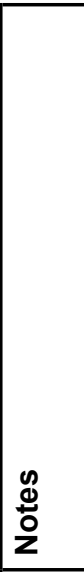 & 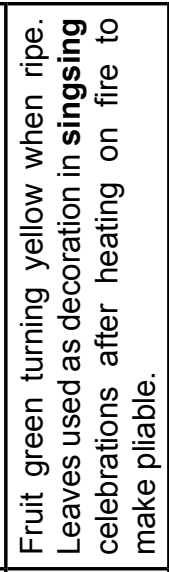 & 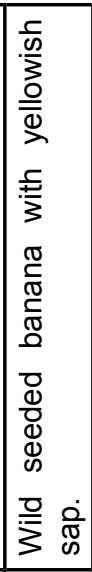 & 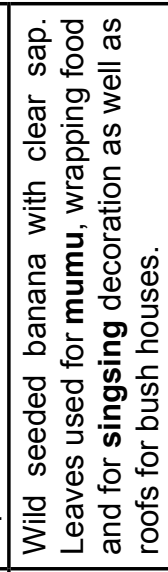 & 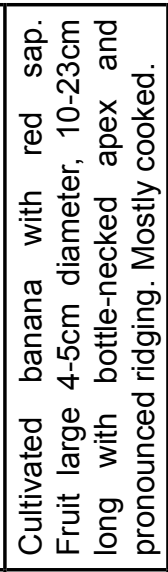 & 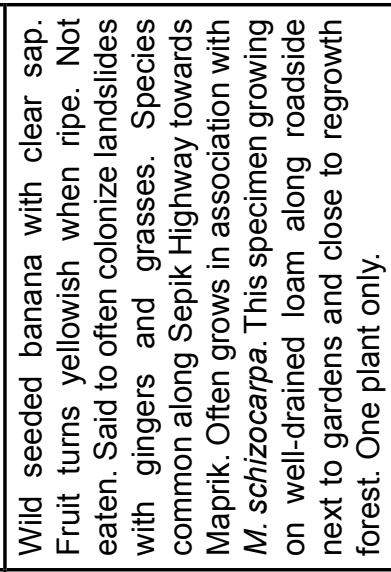 & 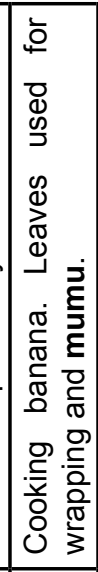 & 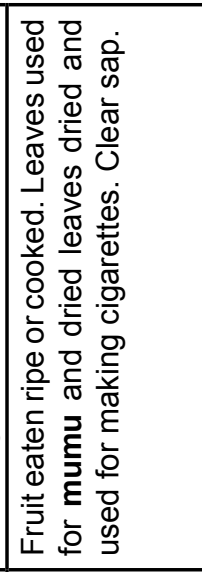 & 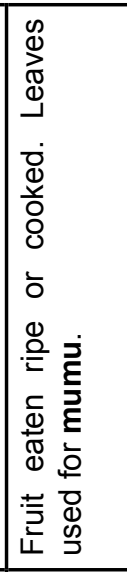 & 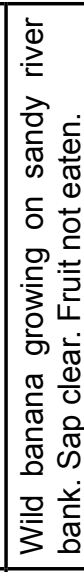 & 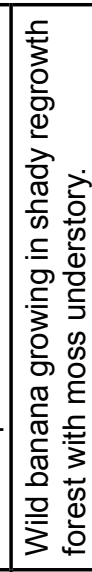 \\
\hline $\begin{array}{l}\frac{0}{0} \\
\frac{\pi}{5} \\
\frac{0}{\pi}\end{array}$ & $\stackrel{\stackrel{\circ}{\Sigma}}{\sum}$ & $\stackrel{\stackrel{\varrho}{\Sigma}}{\sum}$ & $\stackrel{\circ}{\stackrel{(}{\Sigma}}$ & $\stackrel{\circ}{\stackrel{(}{\Sigma}}$ & $\stackrel{\stackrel{\varrho}{\Sigma}}{\sum}$ & $\frac{\frac{c}{50}}{\frac{0}{a}}$ & 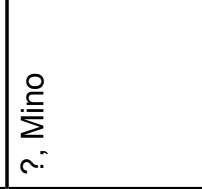 & 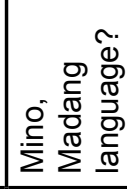 & $\stackrel{\circ}{\stackrel{一}{\Sigma}}$ & 妥 \\
\hline 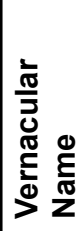 & 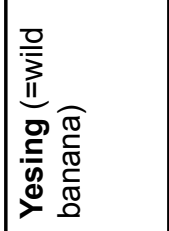 & 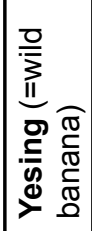 & 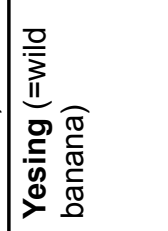 & 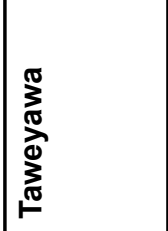 & 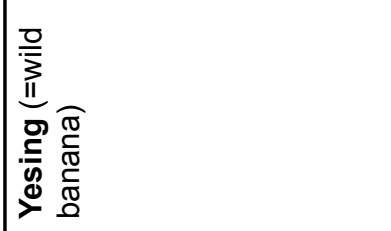 & 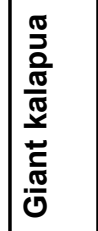 & 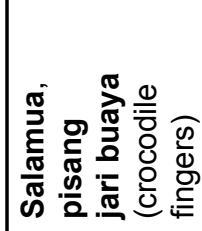 & 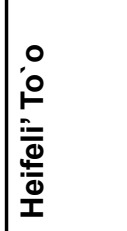 & 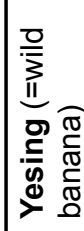 & 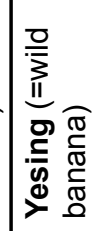 \\
\hline 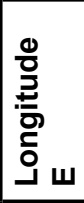 & 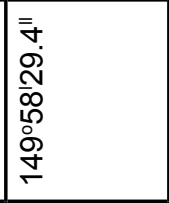 & 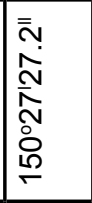 & 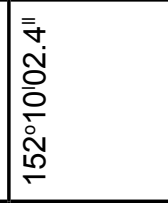 & 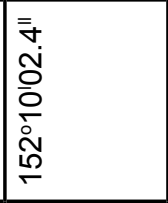 & 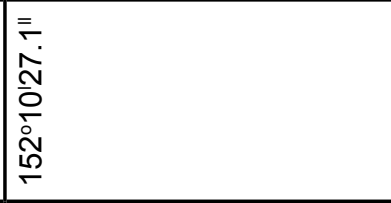 & 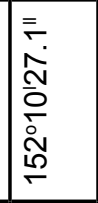 & 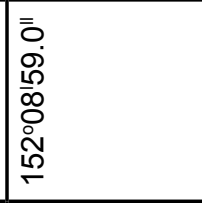 & 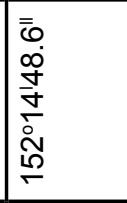 & 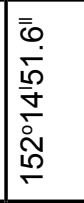 & 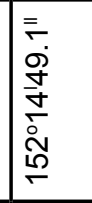 \\
\hline D & 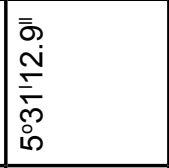 & 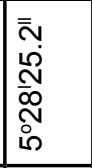 & 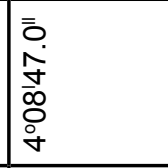 & 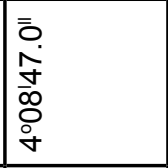 & $\begin{array}{l}\bar{\sigma} \\
\infty \\
\infty \\
1 \\
\infty \\
0 \\
\dot{+} \\
\end{array}$ & 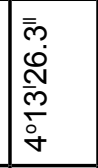 & $\begin{array}{l}\overline{1} \\
\infty \\
\infty \\
10 \\
\infty \\
0 \\
0 \\
\dot{+} \\
\end{array}$ & 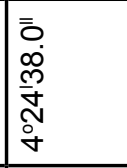 & 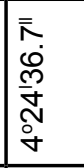 & 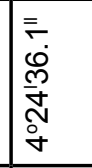 \\
\hline$\frac{0}{P_{0}^{0}}$ & \begin{tabular}{|l}
0 \\
0 \\
$\frac{0}{\pi}$ \\
$\frac{\pi}{3}$ \\
0 \\
0 \\
\end{tabular} & \begin{tabular}{|l}
0 \\
0 \\
$\frac{0}{\pi}$ \\
$\frac{\pi}{3}$ \\
0 \\
0
\end{tabular} & \begin{tabular}{|l}
0 \\
0 \\
$\frac{0}{\pi}$ \\
$\frac{\pi}{3}$ \\
0 \\
0 \\
\end{tabular} & $\begin{array}{l}0 \\
0 \\
\frac{1}{0} \\
\frac{\pi}{3} \\
0 \\
\end{array}$ & \begin{tabular}{|l}
0 \\
0 \\
$\frac{0}{\pi}$ \\
$\frac{\pi}{3}$ \\
0 \\
0 \\
\end{tabular} & $\frac{\xi}{\delta}$ & $\frac{\delta}{\delta}$ & 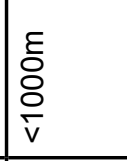 & 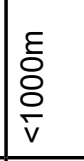 & 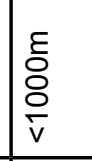 \\
\hline 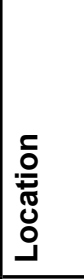 & 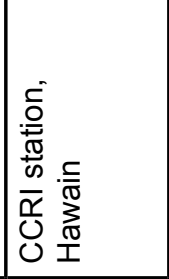 & 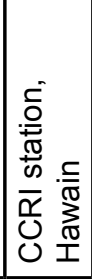 & 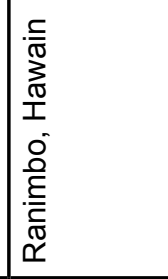 & 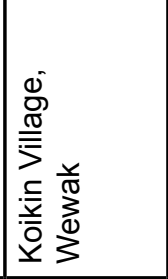 & 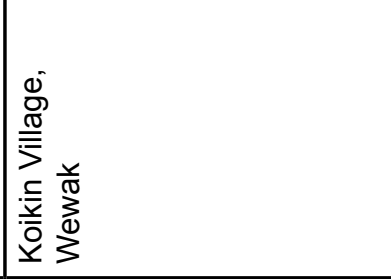 & 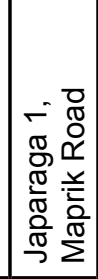 & 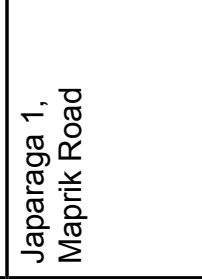 & 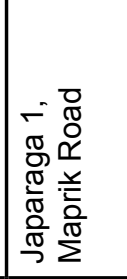 & 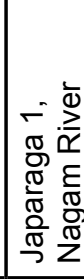 & 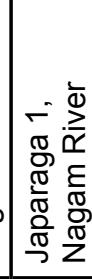 \\
\hline 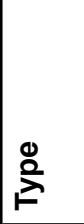 & 安 & 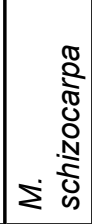 & 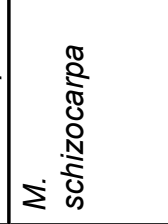 & 漓 & 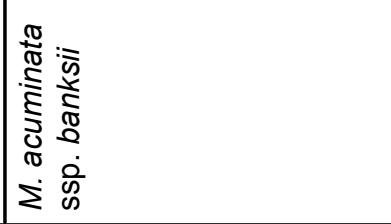 & 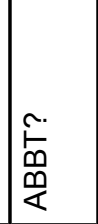 & 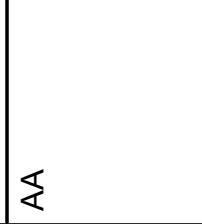 & 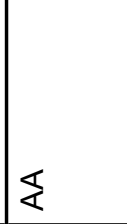 & 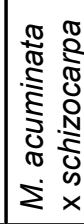 & 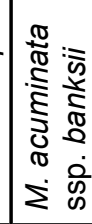 \\
\hline $\begin{array}{l}\stackrel{0}{8} \\
\text { Oे }\end{array}$ & \begin{tabular}{|l}
$\tilde{N}$ \\
$\tilde{w}$
\end{tabular} & $\begin{array}{l}\tilde{M} \\
\tilde{u}\end{array}$ & 志 & W & \begin{tabular}{|l}
0 \\
$\mathscr{D}$ \\
\end{tabular} & \begin{tabular}{|l}
$\hat{c}$ \\
un
\end{tabular} & 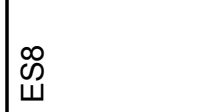 & 怘 & $\frac{\circ}{i}$ & $\underset{\omega}{\bar{\omega}}$ \\
\hline
\end{tabular}




\begin{tabular}{|c|c|c|c|c|c|}
\hline 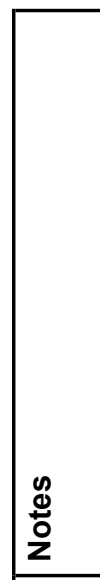 & 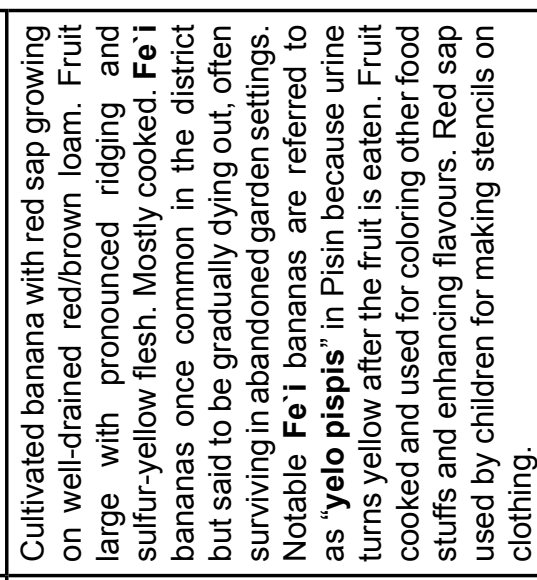 & 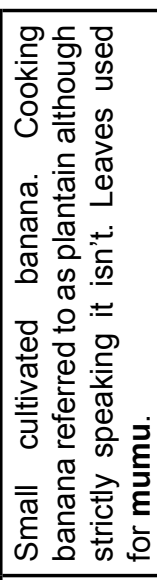 & 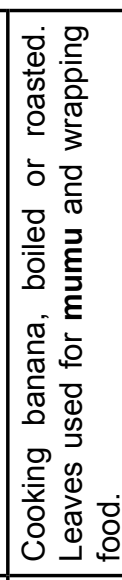 & 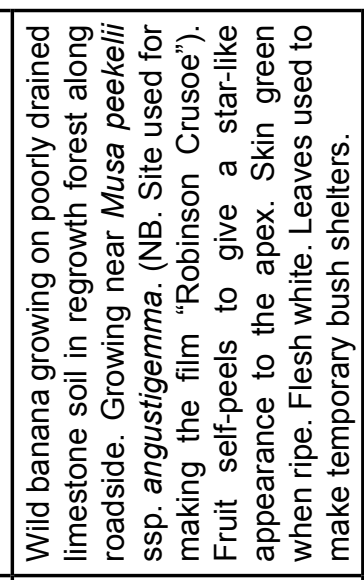 & 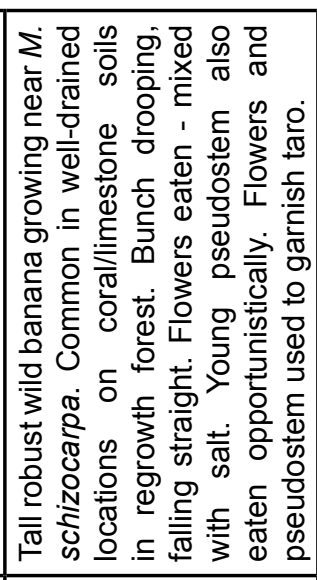 \\
\hline 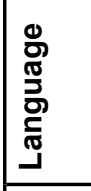 & 安 & \begin{tabular}{|l}
$\frac{\omega}{\omega}$ \\
$\frac{\xi}{2}$ \\
\end{tabular} & 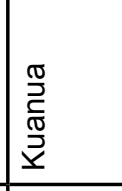 & 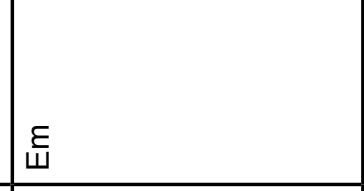 & E् \\
\hline 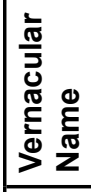 & $\frac{.5}{\frac{1}{10}}$ & 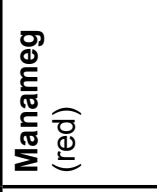 & 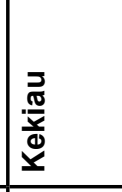 & 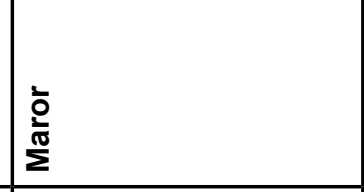 & ڤ̀ \\
\hline 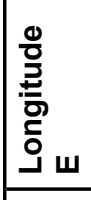 & 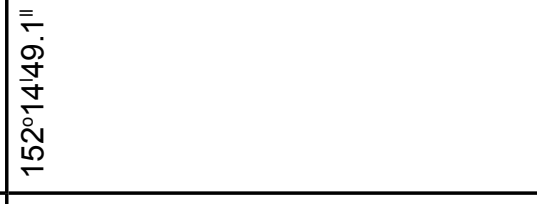 & 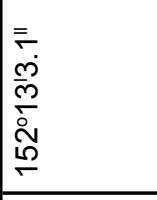 & 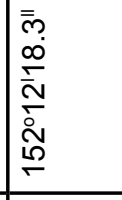 & 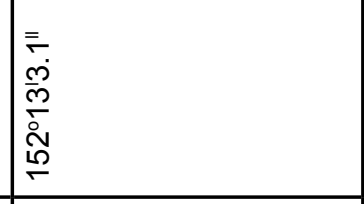 & 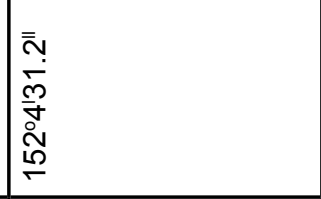 \\
\hline 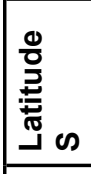 & \begin{tabular}{|l|}
$\bar{\sigma}$ \\
$\dot{0}$ \\
$\tilde{m}$ \\
$\dot{N}$ \\
$\dot{\sigma}$ \\
$\dot{\sigma}$ \\
\end{tabular} & 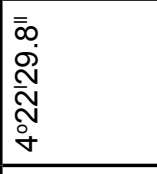 & 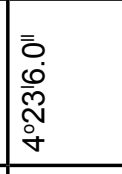 & 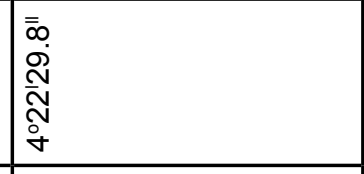 & 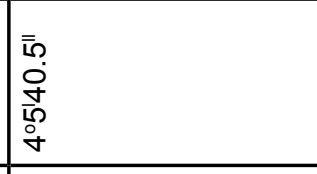 \\
\hline$\frac{8}{\sum^{\frac{3}{2}}}$ & उ & $\begin{array}{l}\frac{1}{8} \\
\frac{0}{5} \\
\frac{\pi}{3} \\
3 \\
9\end{array}$ & 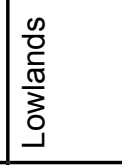 & 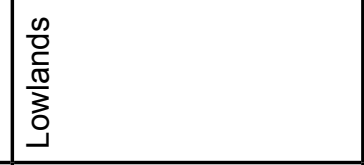 & \begin{tabular}{|l}
$\frac{8}{8}$ \\
$\frac{8}{6}$ \\
$\frac{10}{3}$ \\
3 \\
0
\end{tabular} \\
\hline 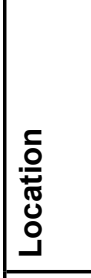 & 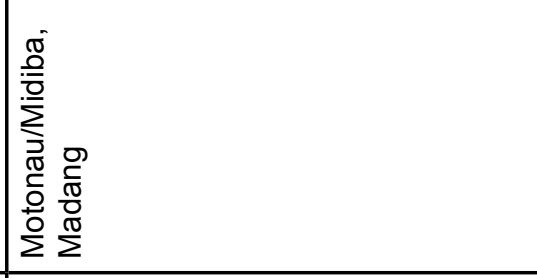 & 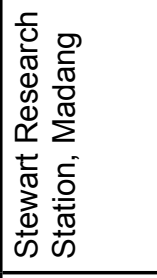 & 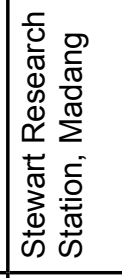 & 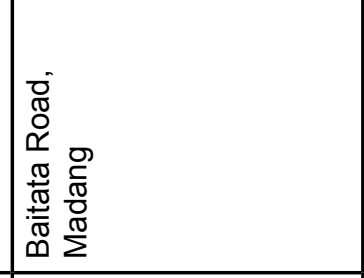 & 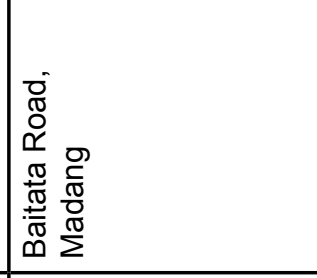 \\
\hline 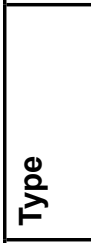 & \begin{tabular}{|l}
- \\
ப் \\
\end{tabular} & 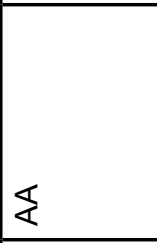 & $\frac{\pi}{4}$ & 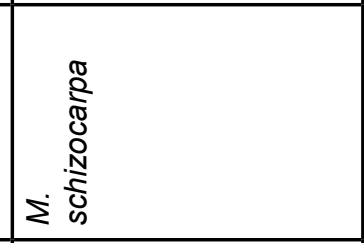 & 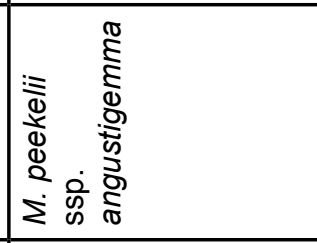 \\
\hline ¿̊ँ & $\Sigma$ & $\sum$ & $\sum^{m}$ & $\sum_{\Sigma}^{*}$ & $\sum^{10}$ \\
\hline
\end{tabular}


Lentfer - Tracing Domestication and Cultivation of Bananas from Phytoliths: 265 An update from Papua New Guinea

\begin{tabular}{|c|c|c|c|c|c|c|c|c|}
\hline 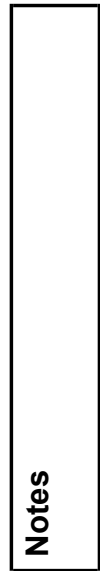 & 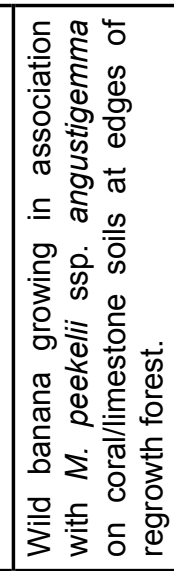 & 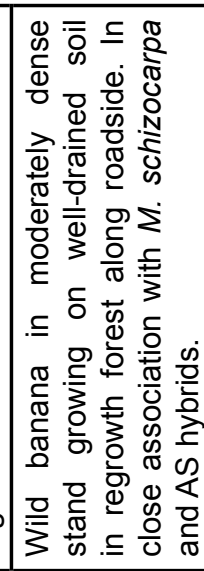 & 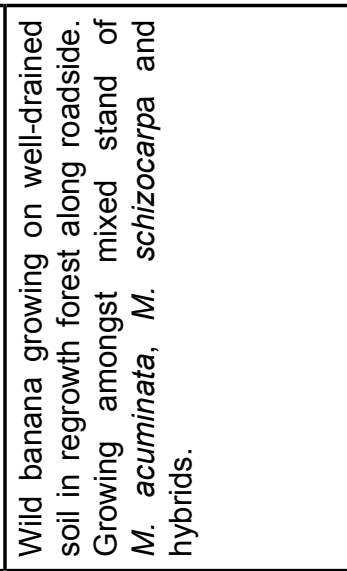 & 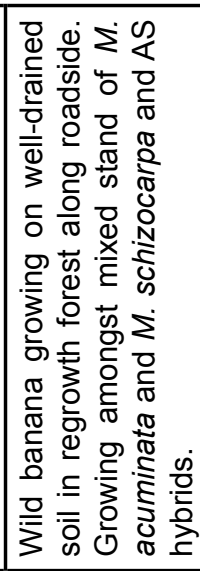 & 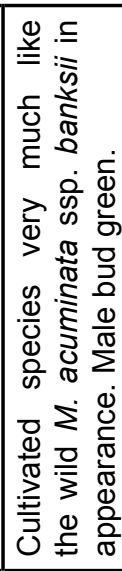 & 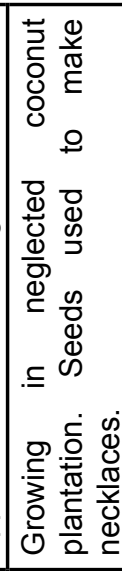 & 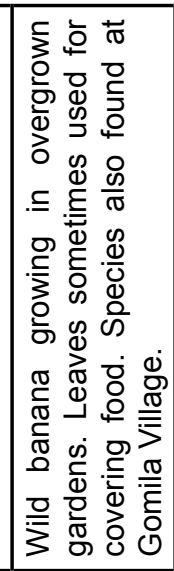 & 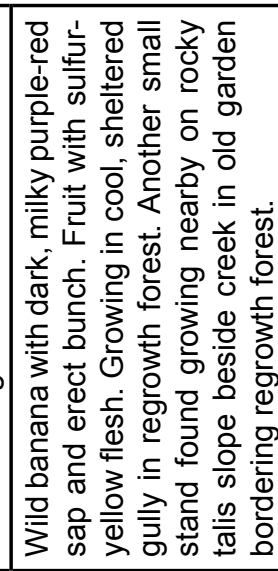 \\
\hline 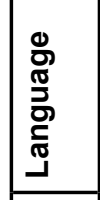 & Е & $\begin{array}{l}\stackrel{c}{\underline{\mathscr{E}}} \\
\end{array}$ & 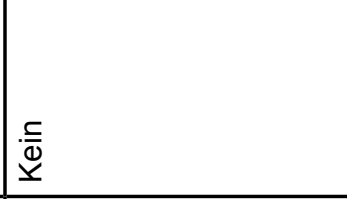 & 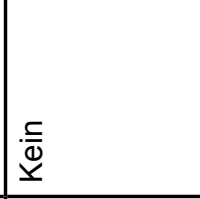 & 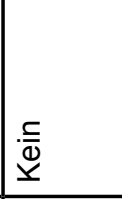 & 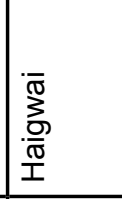 & 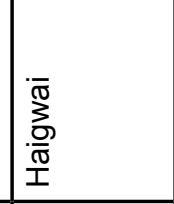 & 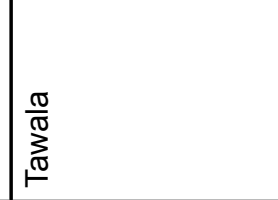 \\
\hline 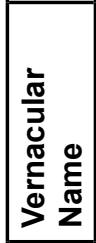 & 爱 & 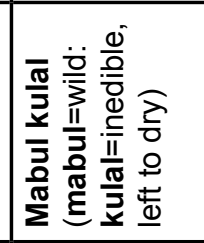 & 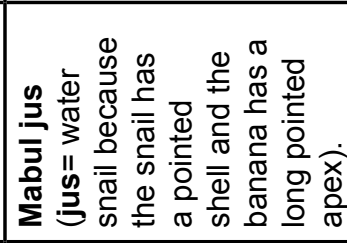 & 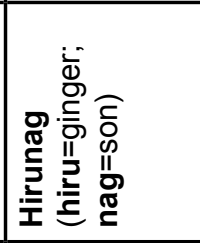 & \begin{tabular}{|l}
$\bar{\Xi}$ \\
$\Phi$ \\
'
\end{tabular} & 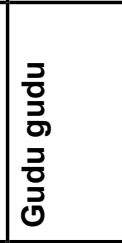 & 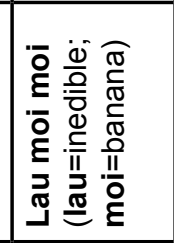 & 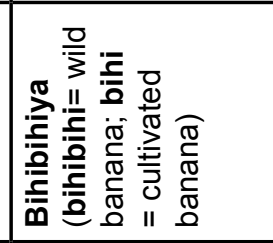 \\
\hline 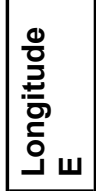 & 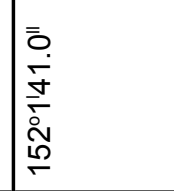 & 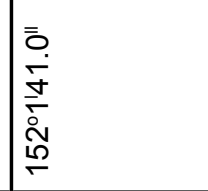 & 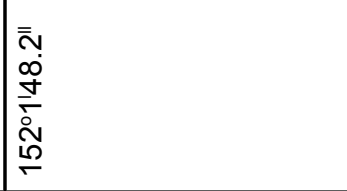 & 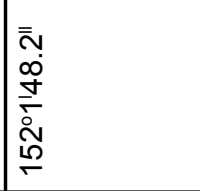 & 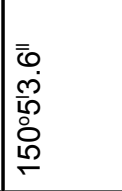 & 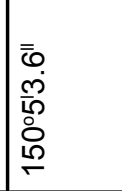 & 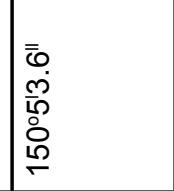 & 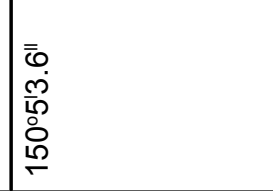 \\
\hline 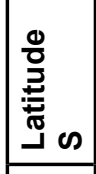 & 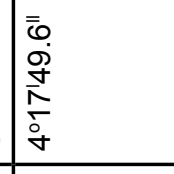 & 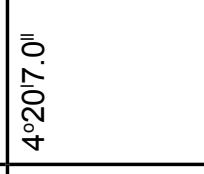 & 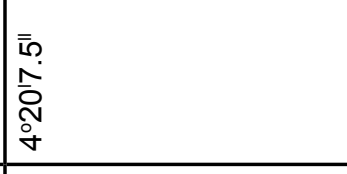 & 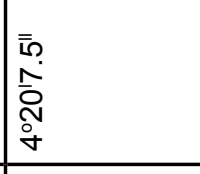 & 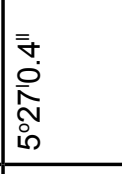 & 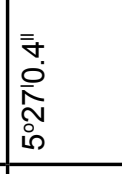 & 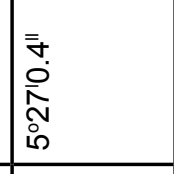 & 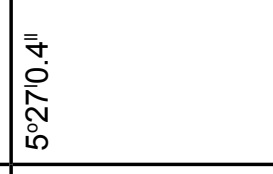 \\
\hline 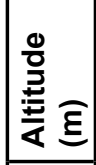 & $\begin{array}{l}0 \\
0 \\
0 \\
\frac{10}{3} \\
3 \\
0 \\
0\end{array}$ & 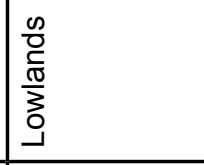 & 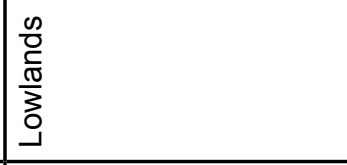 & 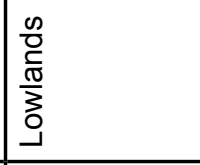 & \begin{tabular}{|l}
0 \\
0 \\
0 \\
$\frac{10}{3}$ \\
3 \\
3 \\
3
\end{tabular} & $\begin{array}{l}0 \\
0 \\
\frac{0}{6} \\
\frac{1}{3} \\
-3 \\
\end{array}$ & 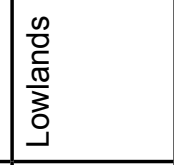 & \begin{tabular}{|l}
0 \\
0 \\
0 \\
$\frac{10}{3}$ \\
3 \\
\\
\end{tabular} \\
\hline 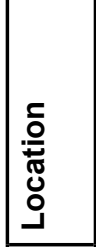 & 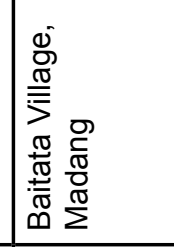 & 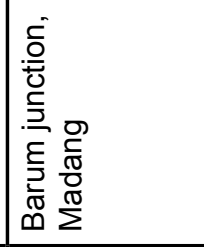 & 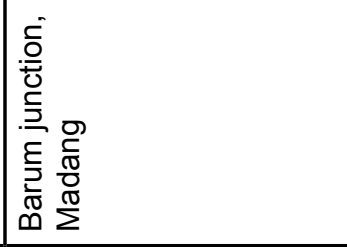 & 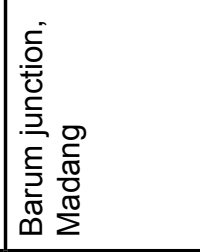 & 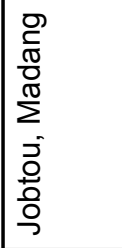 & 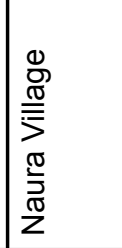 & 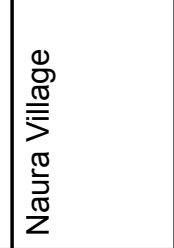 & 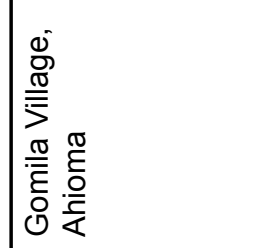 \\
\hline 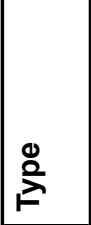 & 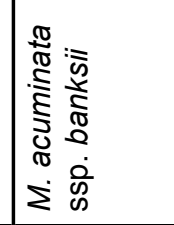 & 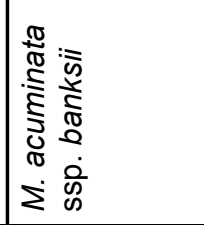 & 18 & 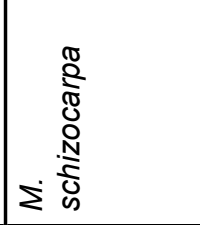 & 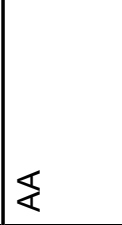 & 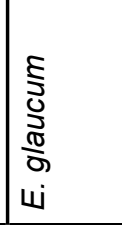 & 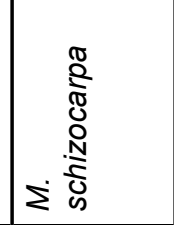 & 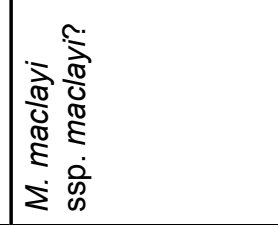 \\
\hline ن & $\stackrel{\oplus}{\Sigma}$ & $\mid \hat{\Sigma}$ & $\sum_{\Sigma}^{\infty}$ & $\frac{\infty}{2}$ & $\left.\right|_{i} ^{\circ}$ & $\stackrel{\infty}{\Sigma}$ & $\stackrel{\tilde{\tilde{a}}}{\Sigma}$ & $\sum_{\substack{\infty \\
\Sigma}}^{\infty}$ \\
\hline
\end{tabular}




\begin{tabular}{|c|c|c|c|c|c|c|c|c|}
\hline $\begin{array}{l}\text { 巳 } \\
\stackrel{0}{0} \\
\text { z }\end{array}$ & 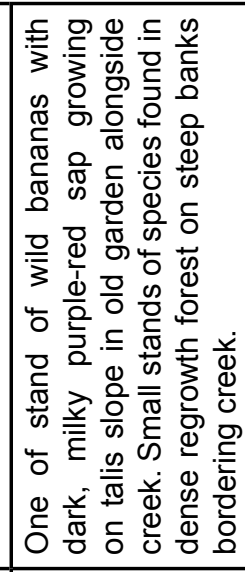 & 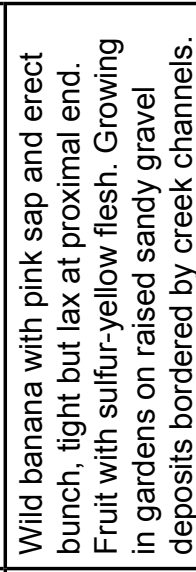 & 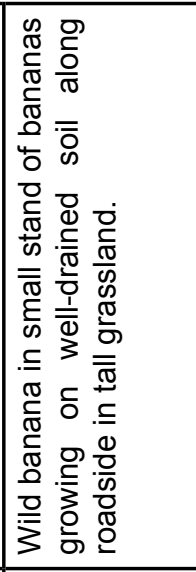 & 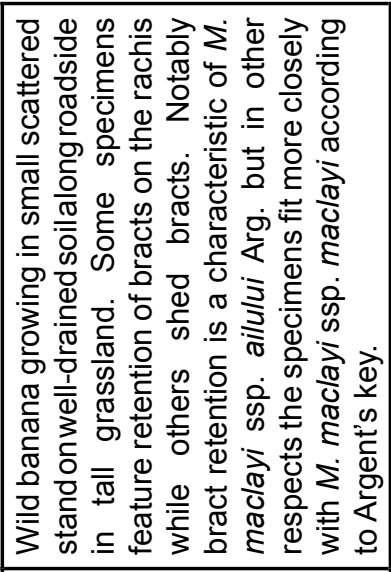 & 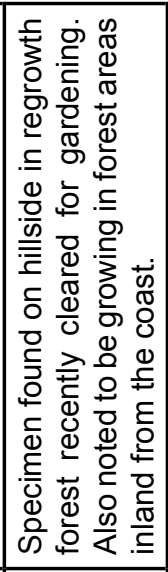 & 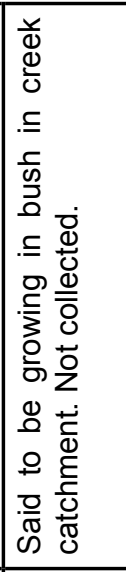 & 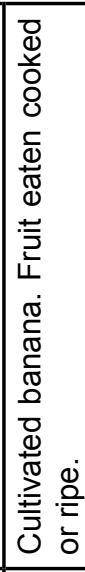 & 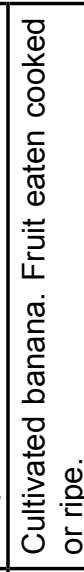 \\
\hline 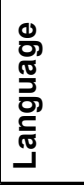 & $\begin{array}{l}\frac{\pi}{\pi} \\
\underset{\pi}{\pi} \\
1\end{array}$ & $\begin{array}{l}\frac{\pi}{\sigma} \\
\underset{\omega}{\infty} \\
\qquad\end{array}$ & 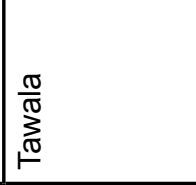 & $\begin{array}{l}\frac{\pi}{\pi} \\
\underset{\pi}{\pi} \\
1\end{array}$ & & 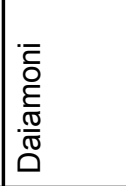 & 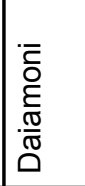 & 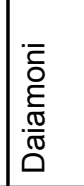 \\
\hline 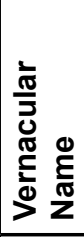 & 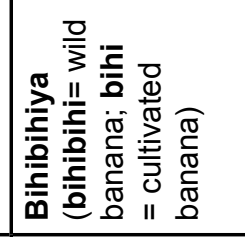 & 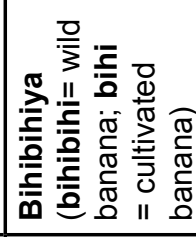 & 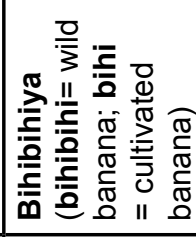 & 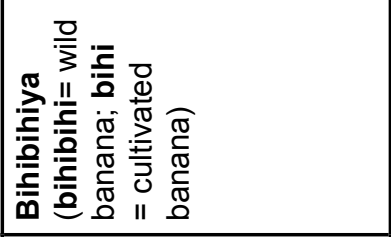 & & 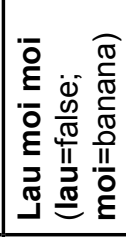 & 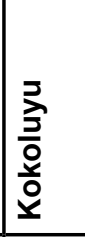 & 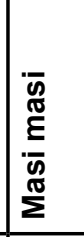 \\
\hline 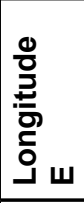 & 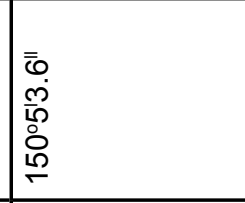 & 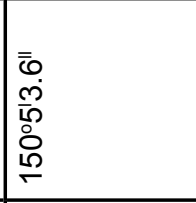 & 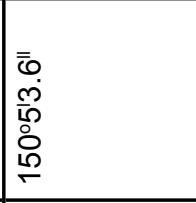 & 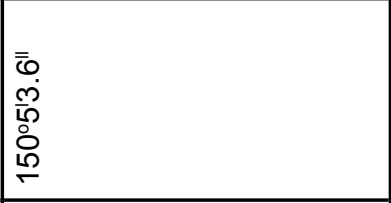 & 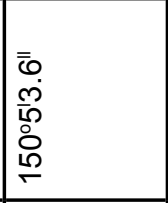 & 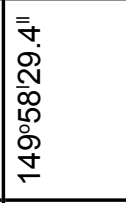 & 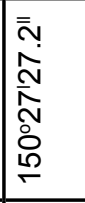 & 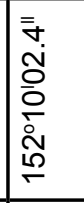 \\
\hline 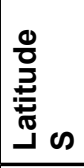 & 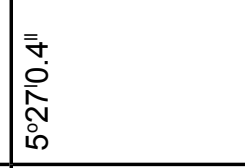 & 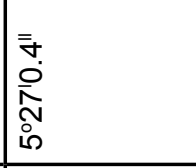 & 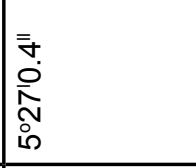 & 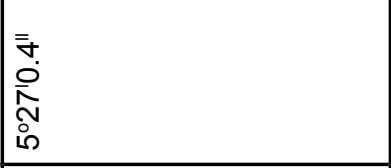 & 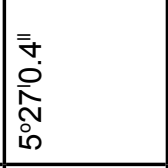 & 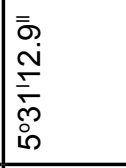 & 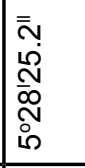 & 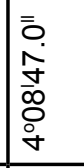 \\
\hline 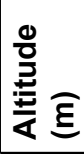 & $\begin{array}{l}\frac{0}{0} \\
\frac{1}{0} \\
\frac{\pi}{3} \\
0 \\
\end{array}$ & \begin{tabular}{|l}
0 \\
0 \\
$\frac{0}{5}$ \\
$\frac{\pi}{3}$ \\
0 \\
0
\end{tabular} & \begin{tabular}{|l}
$\frac{0}{0}$ \\
$\frac{0}{5}$ \\
$\frac{\pi}{3}$ \\
3 \\
0 \\
\end{tabular} & \begin{tabular}{|l}
$\frac{0}{0}$ \\
0 \\
$\frac{1}{\pi}$ \\
$\frac{\pi}{3}$ \\
0 \\
\end{tabular} & \begin{tabular}{|l}
0 \\
0 \\
$\frac{0}{5}$ \\
$\frac{\pi}{3}$ \\
0 \\
0
\end{tabular} & \begin{tabular}{|l}
$\frac{0}{0}$ \\
$\frac{0}{5}$ \\
$\frac{\pi}{3}$ \\
3 \\
0 \\
\end{tabular} & $\begin{array}{l}\frac{0}{0} \\
\frac{1}{5} \\
\frac{\pi}{3} \\
0 \\
\end{array}$ & $\begin{array}{l}0 \\
0 \\
\frac{1}{\pi} \\
\frac{\pi}{3} \\
0 \\
0\end{array}$ \\
\hline 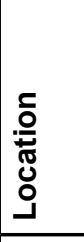 & 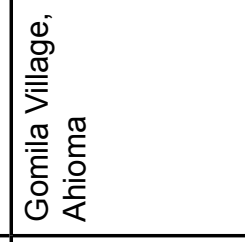 & 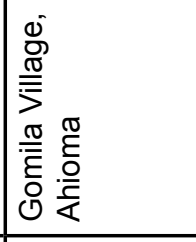 & 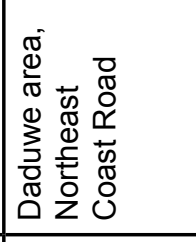 & 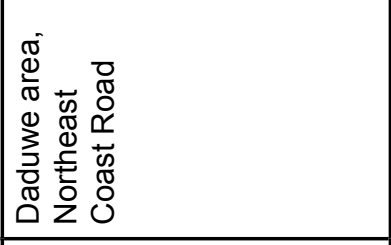 & 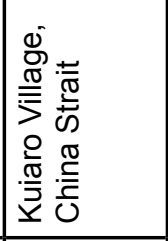 & 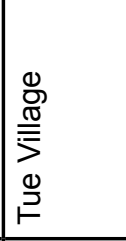 & 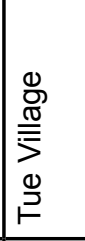 & 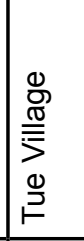 \\
\hline$\stackrel{\Xi}{\gtrsim}$ & 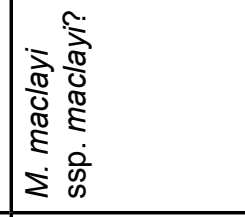 & 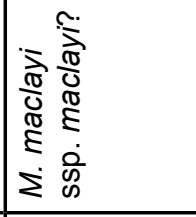 & 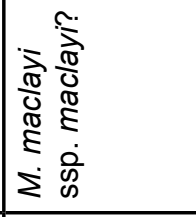 & 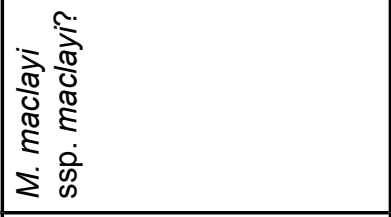 & 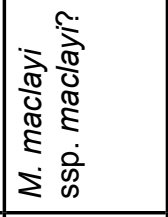 & 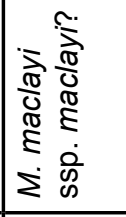 & $\ll$ & 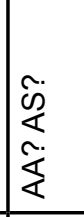 \\
\hline $\begin{array}{l}\frac{0}{0} \\
0 \\
0\end{array}$ & 噮 & 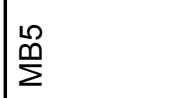 & 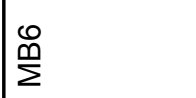 & $\stackrel{\hat{m}}{\Sigma}$ & $\stackrel{\text { Q }}{\Sigma}$ & $\stackrel{\text { Q }}{\Sigma}$ & $\stackrel{\infty}{\stackrel{\infty}{\Sigma}}$ & ஜ̊ \\
\hline
\end{tabular}




\section{Lentfer - Tracing Domestication and Cultivation of Bananas from Phytoliths: 267 An update from Papua New Guinea}

Table 4. Accessions selected for the 'New Guinea Banana Project' analyses.

\begin{tabular}{|c|c|c|c|}
\hline Section & & $\begin{array}{l}\text { Number } \\
\text { analyzed }\end{array}$ & Accession code \\
\hline & \multicolumn{3}{|l|}{ Wild species } \\
\hline \multirow[t]{3}{*}{ Eumusa } & Musa acuminata ssp. banksii & 5 & ES11, ES6, M7, M6, ENB10 \\
\hline & Musa schizocarpa & 5 & ES3, WH5, MB2, M9, ES4 \\
\hline & Musa acuminata ssp. banksii x schizocarpa & 2 & M8, ES10 \\
\hline \multirow[t]{3}{*}{ Australimusa } & Musa peekelii & 2 & M5, NI22 \\
\hline & Musa maclayi & 5 & $\mathrm{~N} 121, \mathrm{MB} 7, \mathrm{MB} 4, \mathrm{MB} 3, \mathrm{MB} 5$ \\
\hline & Musa textilis & 2 & ENB20, WNB6 \\
\hline Ingentimusa & Musa ingens & 3 & $\mathrm{WH} 1, \mathrm{WH} 2, \mathrm{WH} 3$ \\
\hline \multirow[t]{2}{*}{$\mathrm{N} / \mathrm{A}$} & Ensete glaucum & 4 & WNB1, WNB11, MB1, MB1/2 \\
\hline & \multicolumn{3}{|l|}{ Cultivars* } \\
\hline \multirow[t]{6}{*}{ Eumusa } & AA & 5 & $\begin{array}{l}\text { NI12, ENB13, Nari064, } \\
\text { Nari164, MB8 }\end{array}$ \\
\hline & AAA & 5 & $\begin{array}{l}\text { NI8, NariOBB5, NariNB420, } \\
\text { NariOBN14, ENB18 }\end{array}$ \\
\hline & BB? & 1 & ENB12 \\
\hline & $\mathrm{AAB}$ & 5 & NI13, Nari206, Nari146, NI11, NB1 \\
\hline & ABB & 5 & $\begin{array}{l}\text { Nari1047, NariNBG11, NariNBI10, } \\
\text { Nari171, NariNBL20 }\end{array}$ \\
\hline & $A B$ & 1 & ENB17 \\
\hline Australimusa & $\mathrm{TT}\left(\mathbf{F e} \mathbf{i}^{\mathrm{i}}\right)$ & 5 & WH4, ES5, ENB13, M1, ENB14 \\
\hline \multirow{2}{*}{$\begin{array}{l}\text { Eumusa } x \\
\text { Australimusa }\end{array}$} & AAT & 2 & Nari186, ES1? \\
\hline & ABBT? & 1 & ES7 \\
\hline
\end{tabular}

*Genome labels for diploid, triploid and polyploid cultivars: $A=$ acuminata, $B=$ balbisiana, $T=$ Australimusa.

Table 5. Expanded list of categories and attributes used in analysis of volcaniform leaf phytoliths for the 'New Guinea Banana Project'.

\begin{tabular}{|c|c|}
\hline Category & Attribute \\
\hline Base, 3D shape & tabular, blocky, spherical( globose), platy (planar) \\
\hline Base, 2D shape & round, oval, square, rectangle, quadrilateral, boat (oblong), irregular \\
\hline Body length & - \\
\hline Body width & - \\
\hline Body height & short ( $h<1 / 3$ length), medium ( $h=1 / 3$ to $1 / 2$ length), tall ( $h \geq 1 / 2$ length) \\
\hline Crater length & - \\
\hline Crater width & - \\
\hline Crater shape (dorsal view) & round (orbicular), oval, quadrilateral, irregular \\
\hline $\operatorname{Rim}$ & present, absent \\
\hline Rim shape & regular, irregular, skirt (crenate) \\
\hline Sides & straight, convex, concave, straight/concave, concave/convex, straight/convex \\
\hline Body texture & $\begin{array}{l}\text { psilate, granulate, verrucate, nodulose, tuberculate, psilate/verrucate, psilate/granulate, } \\
\text { granulate/verrucate }\end{array}$ \\
\hline Base ornamentation & $\begin{array}{l}\text { absent, short }(<1.25 \mu \mathrm{m}) \text {, medium }(1.25-2.5 \mu \mathrm{m}) \text {, long }(>2.5 \mu \mathrm{m}) \text {, long/tuberculate/ } \\
\text { dendritic, short/medium, medium/long }\end{array}$ \\
\hline
\end{tabular}


ferentiated from phytoliths generated by other plant parts, and the absence of seed phytoliths in an archaeobotanical assemblage can signal presence of seedless/domesticated bananas. However, parthenocarpy and sterility, processes inherent in banana domestication, evolved over several generations, and populations of seeded cultivated diploid bananas are still common in Papua New Guinea. Therefore, it can be difficult to determine the status of bananas (wild or cultivated) in the archaeobotanical record unless there is unequivocal evidence for bananas either being outside their natural range, associated with archaeological and pedogenic features indicative of cultivation, and/or associated with other known cultigens. In the absence of diagnostic seed phytoliths identification is reliant on volcaniform leaf phytoliths. Studies show that some species and cultivars can be discriminated according to crater size and body length and by the presence of certain rare morphotypes. Nevertheless, there is large morphotype variation within and between species; consequently, identification is currently reliant on large sample sizes rarely encountered in fossil assemblages. Therefore, more work is needed to clarify the extent of variability across the geographic range and within different habitats. The 'New Guinea Banana Project' commenced in 2002 with the field collection of samples and resultant analysis of 58 additional accessions. The outcome of these analyses should help to resolve many of the outstanding issues regarding the differentiation of volcaniform leaf phytoliths in the archaeobotanical record, particularly in the Pacific/ Papua New Guinea region.

\section{Acknowledgements}

I am most grateful to the Pacific Science Foundation and the University of Queensland for providing the funding for this project. My sincere thanks to Clair Harris for her assistance. I also thank Jeff Daniells, Bill Boyd and Robin Torrence who assisted with the field collection and the PNG government officials and staff (NARI, DAL, DPI, CCEA and CCRI) in particular Valentine Kambori, Rosa Kambuou and Jeff Wiles for their support and kind assistance with getting the project up and running, and Janet Paofa, Martin Gunther, David Minemba, James Maima, Moses Woruba, Will Akus, Joseph Wasem, Marcel Tokorewaga, Kauwe Murley, Luke Nama, Louis Kurika, March Tovue, Joy Penias, Scola Singit, Nick Eki and Manau Peni for their kind assistance. I also thank Nick Lyons for his kind hospitality and all the people who showed us around their gardens and allowed us to take samples.

\section{Literature Cited}

Argent, G.C.G. 1976. The wild bananas of New Guinea. Notes from the Royal Botanic Garden, Edinburgh 35:77114.

Arnaud, E. \& J.P. Horry. 1997.Editors of Musalogue: A Catalogue of Musa Germplasm. Papua New Guinea Col- lecting Expeditions, 1988-1989. International Network for the Improvement of Banana and Plantain, Montpellier.

Ball, T., L. Vrydaghs, I. van den Houwe, J. Manwaring \& E. De Langhe. 2006. Differentiating banana phytoliths: Wild and edible Musa acuminata and Musa balbisiana. Journal of Archaeological Science 33:1228-1236.

Burkill. I.H. 1935. A Dictionary of the Economic Products of the Malay Peninsula. Crown Agents for the Colonies, London.

Carreel, F, D. González de Léon, P. Lagoda, C. Lanaud, C. Jenny, J.-P. Horry \& H. Tézenas du Montcel. 2002. Ascertaining maternal and paternal lineage within Musa by chloroplast and mitochondrial DNA RFLP analyses. Genome 45:679-692.

Crowther, A. 2009. Investigating Lapita Subsistence and Pottery Use Through Microscopic Residues on Ceramics: Methodological issues, feasibility and potential. Ph.D. Thesis, School of Social Science, Queensland University.

Daniells, J., C. Jenny, D. Karamura \& K. Tomekpe. 2001. Musalogue: A Catalogue of Musa Germplasm. Diversity in the Genus Musa. International Network for the Improvement of Banana and Plantain, Montpellier.

De Langhe, E. \& P. de Maret. 1999. Tracking the banana: its significance in early agriculture. Pp. 377-396 in The Prehistory of Food: Appetites for change. Edited by C. Gosden \& J. Hather. Routledge, London.

De Langhe, E., L. Vrydaghs, P. de Maret, X. Perrier \& T. Denham. 2009. Why bananas matter: An introduction to the history of banana domestication. Ethnobotany Research and Applications 7:165-177.

Denham, T.P. \& M. Donohue. 2009. Pre-Austronesian dispersal of banana cultivars west from New Guinea: linguistic relics from eastern Indonesia. Archaeology in Oceania 44:18-28.

Denham, T.P., S.G. Haberle, C.J. Lentfer, R. Fullagar, J. Field, M. Therin, N. Porch \& B. Winsborough. 2003. Origins of agriculture at Kuk Swamp in the Highlands of New Guinea. Science 301:189-193.

Donohue, M. \& T.P. Denham. 2009. Banana (Musa spp.) domestication in the Asia-Pacific region: Linguistic and archaeobotanical perspectives. Ethnobotany Research and Applications 7:293-332.

Horrocks, M., S. Bedford \& M. Spriggs. In press. A short note on banana (Musa) phytoliths in Lapita, immediately post-Lapita and modern period archaeological deposits from Vanuatu. Journal of Archaeological Science. 


\section{Lentfer - Tracing Domestication and Cultivation of Bananas from Phytoliths: 269 An update from Papua New Guinea}

Horrocks, M., S. Bulmer \& R.O. Gardner 2007. Plant microfossils in prehistoric archaeological deposits from Yuku rock shelter, Western Highlands, Papua New Guinea. Journal of Archaeological Science 35:290-301.

Jarret, R.L., N. Gawel, A. Whittemore \& S. Sharrock. 1992. RFLP-based phylogeny of Musa species in Papua New Guinea. Theoretical and Applied Genetics 84:579-584.

Kennedy, J. 2008. Pacific bananas: Complex origins, multiple dispersals? Asian Perspectives 47:75-94.

Kennedy, J. 2009. Bananas and people in the homeland of genus Musa: Not just pretty fruit. Ethnobotany Research and Applications 7:179-197.

Lebot, V. 1999. Biomolecular evidence for plant domestication in Sahul. Genetic Resources and Crop Evolution 46:619-628.

Lejju, B.J., P. Robertshaw \& D. Taylor. 2006. Africa's earliest bananas? Journal of Archaeological Science 33:102113.

Lentfer, C.J. 2003a. Tracing Antiquity of Banana Cultivation in Papua New Guinea: Report on collection of modern reference material from Papua New Guinea in 2002. Unpublished report prepared for the Pacific Biological Foundation, Sydney, NSW, Australia.

Lentfer, C.J. 2003b. Plants, People and Landscapes in Prehistoric Papua New Guinea. A compendium of phytolith (and starch) analyses. Ph.D. Thesis, Southern Cross University, Lismore, N.S.W., Australia.

Lentfer, C.J. 2009. Going bananas in Papua New Guinea: A preliminary study of starch granule morphotypes in Musaceae fruit. Ethnobotany Research and Applications 7:217-238.

Lentfer, C.J. \& R.C. Green. 2004. Phytoliths and the evidence for banana cultivation at the Lapita Reber-Rakival site on Watom Island, Papua New Guinea. Pp. 75-88 in A Pacific Odyssey: Archaeology and anthropology in the Western Pacific. Papers in Honour of Jim Specht. Records of the Australian Museum 29. Edited by V. Attenbrow \& R. Fullagar. Australian Museum, Sydney.

Lentfer, C.J., C. Pavlides, R. Fullagar \& J. Specht. 2008. Subsistence and Environmental History in Central New Britain, Papua New Guinea: Combining phytoliths, macrofossils and use-wear/residue studies. Paper presentation at the Australian Archaeological Conference, Noosa, Qld. December 2008.

MacDaniels, L.H. 1947. A Study of the Fe 'i Banana and its Distribution with Reference to Polynesian Migrations. Ber- nice P. Bishop Museum Bulletin 190. Bernice P. Bishop Museum, Honolulu.

Madella, M., A. Alexandre \& T. Ball. 2005. International code for phytolith nomenclature 1.0. Annals of Botany 96:253-260.

Mbida, C.M., H. Doutrelepont, L. Vrydaghs, R.L. Swennen, R.J. Swennen, H. Beeckman, E. De Langhe \& P. de Maret. 2001. First archaeological evidence of banana cultivation in central Africa during the third millennium before present. Vegetation History and Archaeobotany 10:1-6.

Mbida, C.M., H. Doutrelepont, L. Vrydaghs, R.L. Swennen, R.J. Swennen, H. Beeckman, E. De Langhe, \& P. de Maret. 2004. Yes, there were bananas in Cameroon more than 2000 years ago. InfoMusa 13:40-42.

Neumann, K. \& E. Hildebrand 2009. Early bananas in Africa: The state of the art. Ethnobotany Research and Applications 7:353-362.

Perrier, X., F. Bakry, F. Carreel, Ch. Jenny, J.-P. Horry, V. Lebot \& I. Hippolyte. 2009. Combining biological approaches to shed light on evolution of edible bananas. Ethnobotany Research and Applications 7:199-216.

Pollefeys, P., S. Sharrock \& E. Arnaud. 2004. Preliminary Analysis of the Literature on the Distribution of Wild Musa Species using MGIS and DIVA-GIS. International Network for the Improvement of Banana and Plantain, Montpellier, and International Plant Genetic Resources Institute, Rome.

Sharrock, S. 1990. Collecting Musa in Papua New Guinea. Pp. 140-157 in Identification of Genetic Diversity in the Genus Musa: Proceedings of an International Workshop held at Los Baños, Philippines, 5-10 September 1988. Edited by R.L. Jarret. International Network for the Improvement of Banana and Plantain, Montpellier.

Simmonds, N.W. 1959. Bananas. Longmans, Green and Co, London.

Simmonds, N.W. 1962. The Evolution of the Bananas. Longmans, Green and Co, London.

Spriggs, M. 1996. What is Southeast Asian about Lapita? Pp. 324-348 in Prehistoric Mongoloid Dispersals. Edited by T. Akazawa \& E. Szathmary. Oxford University Press, Oxford.

Valmayor, R.V. 2001. Classification and characterization of Musa exotica, $M$. alinsanaya and $M$. acuminata ssp. errans. InfoMusa 10:35-39.

Vrydaghs, L., T. Ball, H. Volkaeart, I. van den Houwe, J. Manwaring \& E. De Langhe. 2009. Differentiating the vol- 
caniform phytoliths of bananas: Musa acuminata. Ethnobotany Research and Applications 7:239-246.

Wilson, S.M. 1985. Phytolith analysis at Kuk, an early agricultural site in Papua New Guinea. Archaeology in Oceania 20:90-97.
Wong, C., R. Kiew, J.P. Loh, L.H. Gan, O. Set, S.K. Lee, S. Lum \& Y.Y. Gan. 2001. Genetic diversity of the wild banana Musa acuminata Colla in Malaysia as evidenced by AFLP. Annals of Botany 88:1017-1025. 